\title{
Thomas-Fermi-Dirac-von Weizsäcker hydrodynamics in laterally modulated electronic systems
}

\author{
B.P. van Zyl and E. Zaremba \\ Department of Physics, Queen's University, \\ Kingston, Ontario, Canada K' ${ }^{7}$ LN6
}

(August 19, 2018)

\begin{abstract}
We have studied the collective plasma excitations of a two-dimensional electron gas with an arbitrary lateral charge-density modulation. The dynamics is formulated using a previously developed hydrodynamic theory based on the Thomas-Fermi-Dirac-von Weizsäcker approximation. In this approach, both the equilibrium and dynamical properties of the periodically modulated electron gas are treated in a consistent fashion. We pay particular attention to the evolution of the collective excitations as the system undergoes the transition from the ideal two-dimensional limit to the highly-localized one-dimensional limit. We also calculate the power absorption in the long-wavelength limit to illustrate the effect of the modulation on the modes probed by far-infrared (FIR) transmission spectroscopy.
\end{abstract}

PACS: 73.20.Dx, 73.20.Mf, 73.23.-b 


\section{INTRODUCTION}

Two-dimensional electronic systems, as found in single heterojunction interfaces or in more complex structures such as quantum wells, have been studied intensively because of their fundamental interest and for their potential application 1 - In particular, the twodimensional electron gas (2DEG) with a spatially periodic modulation of its equilibrium density has attracted considerable attention both theoretically $\mathrm{E}^{-19}$ and experimentally 2028. One way of achieving these systems 20 is to apply a voltage between a patterned gate and the 2DEG, thereby inducing a one-dimensional periodic modulation of the density. As the gate voltage is increased, electrons tend to 'pool' near the minima of the modulating potential and, in the limit of strong modulation, the system transforms into a periodic array of 1D quantum wires. It is clear that the restricted motion of the electrons along the wires will have a profound effect on the character of the plasma excitations in the system. Our main focus in this paper will be to study the evolution of these collective modes with increasing modulation of the 2DEG, and in particular, the crossover from 2D to 1D behaviour.

The theoretical approaches commonly used to discuss collective excitations in chargedensity-modulated systems can be classified broadly as being either hydrodynamic in nature 10.30.31, or based on the random phase approximation (RPA) 6 11 $15 \cdot 17$ 19. The hydrodynamic approaches are appealing because of their relative mathematical and computational simplicity. Although they vary in their level of sophistication, they all have in common the objective of describing the dynamics of the electronic system in terms of a closed set of equations for the density and velocity fields. At the simplest level of approximation 10,10 , the current density in the electron fluid is determined by a local conductivity, proportional to the equilibrium electron density. Poisson's equation is then used to relate the electric field driving the current to the fluctuating electron density. Rather than explicitly determining the equilibrium properties of the electronic system, the equilibrium density is simply chosen to have some physically reasonable form. We shall refer to such theories as 'classical' in that no explicit quantum mechanical aspects are included in either the equilibrium or dynamical properties.

Although the classical treatment is useful for obtaining a qualitative understanding of the collective modes in a modulated 2DEG, it has the shortcoming of not being able to account for nonlocal effects (e.g. in the magneto-conductivity tensor) which become important with increasing magnitude of the plasmon wavevector. Nonlocal effects in the plasmon dispersion can be included in the hydrodynamic theory through the introduction of an electronic compressibility 30 . In this way some information about the quantum mechanical equation of state can be built into the theory. In this respect, these theories can be referred to as 'semiclassical'. The RPA on the other hand, provides a fully quantum mechanical description of both the equilibrium and dynamical behaviour. However, being more general, the computational demands for its implementation are significantly greater. Furthermore, since it includes both single-particle and collective aspects, it is sometimes difficult to cleanly identify excitations which are predominantly collective in nature 29 . For both of these reasons, it is still worthwhile having available hydrodynamic based theories.

In this paper, we investigate the collective response of a modulated 2DEG using the Thomas-Fermi-Dirac-von Weizsäcker (TFDW) hydrodynamics previously developed to treat magnetoplasma excitations in three-dimensional parabolic wells 32 . 33 and electron rings 34. 
One of the primary virtues of this approach is that it is based on a reasonably accurate description of the ground state properties of the electronic system, which are determined self-consistently from the minimization of the TFDW energy functional. Thus, unlike most earlier hydrodynamic treatments, the form of the equilibrium density is not chosen arbitrarily. Perturbations of the system away from the equilibrium state generate internal forces which drive the system back towards equilibrium. These forces are consistently included in the TFDW hydrodynamic equations used to describe the dynamics of the system. Although somewhat more sophisticated, the present approach nevertheless retains much of the mathematical simplicity of the usual hydrodynamic theories.

Our paper is organized as follows. In Sec. II we determine the equilibrium properties of a periodically modulated 2DEG within the TFDW approximation. Sec. III then provides the mathematical formalism needed to study the dynamics of the collective modes and includes a derivation of the power absorption which is typically measured in infrared transmission experiments. In Sec. IV, we consider in detail the crossover from 2D to 1D behavior in the periodically-modulated 2DEG, and finally in Sec. D, we present our concluding remarks.

\section{EQUILIBRIUM PROPERTIES}

The fabrication of mesoscopic devices often begins with a 2DEG in metal-oxidesemiconductor (MOS) structures or epitaxially grown heterojunctions. In these systems, the strong confinement of the electrons in the direction normal to the interface leads to a discrete spectrum of quantized subband states, while motion in the lateral dimensions is essentially free. In the limit that electronic excitations take place within only a single subband, the system can be idealized as strictly two-dimensional with no spatial extent in the normal direction. In this situation, the electronic density has the form $n(x, y, z)=n(x, y) \delta(z)$, where the planar density $n(x, y)$ is defined by whatever additional lateral confining potential $v_{\text {ext }}(x, y)$ is imposed on the system.

To determine the equilibrium density distribution $n(x, y)$, we shall make use of the Thomas-Fermi-Dirac-von Weiszäcker energy functional:

$$
\begin{aligned}
E[n] & =\int d \mathbf{r}\left[C_{1} n^{2}+C_{2} \frac{|\nabla n(\mathbf{r})|^{2}}{n(\mathbf{r})}-C_{3} n^{3 / 2}\right] \\
& +\frac{1}{2} \int d \mathbf{r} \int d \mathbf{r}^{\prime} \frac{n(\mathbf{r}) n\left(\mathbf{r}^{\prime}\right)}{\left|\mathbf{r}-\mathbf{r}^{\prime}\right|}+\int d \mathbf{r} v_{\mathrm{ext}}(\mathbf{r}) n(\mathbf{r}) .
\end{aligned}
$$

The first term in (1) is the Thomas-Fermi kinetic energy, the second term is the von Weizsäcker correction to the kinetic energy, and the third term is the Dirac local exchange energy 35 . For simplicity, we neglect any correlation contribution. The coefficients in (11) are given by (atomic units, $e^{2} / \epsilon=m^{*}=\hbar=1$, are used throughout)

$$
C_{1}=\frac{\pi}{2}, \quad C_{2}=\frac{\lambda_{w}}{8}, \quad C_{3}=\frac{4}{3} \sqrt{\frac{2}{\pi}} .
$$

In the von Weizsäcker coefficient $C_{2}$, the parameter $\lambda_{w}$ is chosen to have the value 0.25 , which was found in other applications 36 to provide the best agreement between the TFDW and full 
density functional theory (DFT) calculations. The last two terms in Eq. (11) are the Hartree self-energy of the electrons and the interaction with the external potential, respectively.

The equilibrium properties are obtained by finding the variational minimum of Eq. (间). This leads to the Euler-Lagrange equation

$$
\frac{\delta E[n]}{\delta n(\mathbf{r})}-\mu=0
$$

where the Lagrange multiplier (chemical potential) $\mu$ serves to fix the total number of electrons, $N$. By introducing a wave function according to the prescription $n(\mathbf{r})=\psi^{2}(\mathbf{r})$, a straightforward calculation yields

$$
-\frac{\lambda_{w}}{2} \nabla^{2} \psi(\mathbf{r})+v_{\mathrm{eff}}(\mathbf{r}) \psi(\mathbf{r})=\mu \psi(\mathbf{r})
$$

where the effective potential is given by

$$
v_{\text {eff }}(\mathbf{r})=2 C_{1} \psi^{2}(\mathbf{r})-\frac{3}{2} C_{3} \psi(\mathbf{r})+\phi(\mathbf{r})+v_{\text {ext }}(\mathbf{r}) .
$$

Here, $\phi(\mathbf{r})=\int d \mathbf{r}^{\prime} n\left(\mathbf{r}^{\prime}\right) /\left|\mathbf{r}-\mathbf{r}^{\prime}\right|$ is the electrostatic potential arising from the electronic density $n(\mathbf{r})$. Eq. (4) is a nonlinear equation in $\psi(\mathbf{r})$, and must be solved self-consistently. The required solution to Eq. (四) is the ground state wave function, hereafter denoted by $\psi_{0}$, and the ground state energy eigenvalue defines $\mu$.

This formulation of the equilibrium properties of the electronic system is quite general in the sense that the physical situation is completely specified by the form of the external potential. For the case of $1 \mathrm{D}$ modulation, the potential $v_{\text {ext }}(x)$ will be assumed to be a smooth, periodic function of $x$ with period $a$. This potential can of course be represented as a Fourier series, but we shall consider only the simplest form

$$
v_{\text {ext }}(x)=-V_{0} \cos \left(\frac{2 \pi x}{a}\right)
$$

having a single Fourier component. This choice is sufficient to investigate the important effects of modulation on the properties of a 2DEG, and has been applied successfully by other workers $14,15,28$ to model laterally microstructured field-effect devices used in FIR experiments.

The translational invariance of the system along the $y$-direction, together with the periodicity of the external confining potential, implies that the solution to Eq. (耳) will only depend on $x$ and will have the property that $\psi_{0}(x+a)=\psi_{0}(x)$. Thus, we can restrict our calculation to the unit cell $x \in[-a / 2, a / 2]$. For potentials such as (6) with inversion symmetry about the center of the cell, the desired solutions are those satisfying the boundary conditions

$$
\psi_{0}^{\prime}\left( \pm \frac{a}{2}\right)=0
$$

To complete the determination of $\psi_{0}(x)$, we must finally impose the normalization condition

$$
\frac{1}{a} \int_{-a / 2}^{a / 2} d x\left|\psi_{0}(x)\right|^{2}=\bar{n}_{2 D}
$$


where $\bar{n}_{2 D}$ is the average electron density in the modulated 2DEG.

Before discussing the nature of the ground state solution to Eq. (4), we first note some technical points involved in the calculation. As is typical of DFT schemes, the solutions are obtained using an iterative procedure, and issues of numerical stability and convergence therefore arise. The usual source of numerical instabilities is the long-ranged nature of the Coulomb interaction. In the course of iterating, a small deviation of the charge density from its true value leads to a change in the effective potential that tends to induce an overcompensating screening charge on the next iteration. In our particular case, this results in an oscillation of the charge between the central and outer regions of the unit cell, which inhibits the desired convergence of the iterative procedure. This problem can be remedied by a combination of two strategies. First, we introduce an algebraic mixing of the effective potential

$$
v_{\mathrm{eff}}^{(i+1)}=\alpha v_{\mathrm{eff}}\left[n^{(i)}\right]+(1-\alpha) v_{\mathrm{eff}}^{(i)}
$$

where $v_{\text {eff }}^{(i)}$ is the effective potential on the $i$-th iteration, $n^{(i)}$ is the density generated by this potential and $v_{\text {eff }}\left[n^{(i)}\right]$ is the effective potential that this density would lead to. The mixing parameter $\alpha$ lies between zero and one and reducing its value reduces the change in the potential from one iteration to the next. This by itself will eliminate the instability in some cases, as for example when the modulation periods are relatively small. However, to access the physically relevant modulation periods (100 nm $<a<1500 \mathrm{~nm}$ in GaAs), an extremely small value of $\alpha$ would have to be used, which results in an unacceptably slow rate of convergence. This problem can be overcome by means of a second strategy in which the long-range nature of the Coulomb potential is effectively screened. The screening is conveniently formulated in Fourier space using the algorithm

$$
\phi^{(i+1)}(\mathbf{q})=\frac{2 \pi n^{(i)}(\mathbf{q})}{Q}+\left(1-\frac{q}{Q}\right) \phi^{(i)}(\mathbf{q})
$$

where $\phi(\mathbf{q})$ and $n(\mathbf{q})$ are the 2D Fourier transforms of the electrostatic potential and 2D electron density, respectively, $Q=\sqrt{q^{2}+\kappa^{2}}$, and $\kappa$ is an artificial screening parameter. One can check that the first term on the right hand side of (10), in which the density acts as a source, leads to an exponentially decaying potential in real space. Nevertheless, at selfconsistency, $\phi^{(i+1)}(\mathbf{q})=\phi^{(i)}(\mathbf{q})=\phi(\mathbf{q}) \equiv 2 \pi n(\mathbf{q}) / q$, which is just the Fourier transform of the actual Coulomb potential $\phi(\mathbf{r})$. By choosing $\kappa$ of order unity, we were able to obtain convergence in all cases of interest with $\alpha>0.005$. Although these values of $\alpha$ are still rather small, they provided a rate of convergence which was significantly better than that achieved with the algebraic mixing scheme alone.

The solution to Eq. (田) is shown in Fig. 1 for a range of potential modulation amplitudes. It is useful to represent these amplitudes in the dimensionless form $V_{0} / E_{f}$, where $E_{f}=\frac{1}{2} k_{f}^{2}$ is the Fermi energy of the uniform 2DEG and the Fermi wavevector $k_{f}$ is defined by $k_{f}^{2} \equiv$ $2 \pi \bar{n}_{2 D}$. With an application to GaAs in mind, typical densities are in the range $10^{11}$ $10^{12} \mathrm{~cm}^{-2}$. Using the effective mass $m^{*}=0.067 m_{e}$ and static dielectric constant $\epsilon=13.0$ of GaAs, the atomic unit of length is the effective Bohr radius $a_{0}^{*}=103 \AA$, and the unit of energy is the effective Rydberg $R y^{*}=e^{2} / 2 \epsilon a_{0}^{*}=5.4 \mathrm{meV}$. In these units, a density of $10^{11} \mathrm{~cm}^{-2}$ is approximately $0.1\left(a_{0}^{*}\right)^{-2}$; this is the value of the average density used to generate 
the density distributions in Fig. 1. The equilibrium densities in this figure span the range from weak to moderate to strong modulation, and illustrate the way in which the cellboundary density decreases with increasing values of $V_{0}$. In the extreme localized limit, the system consists of isolated quantum wires, each of which is confined within an approximately harmonic potential well of curvature $2 \pi^{2} V_{0} / a^{2}$. It can be seen that the density in this limit is similar to the semi-circular profile found in the classical electrostatic approximation 16 , and only deviates significantly from this form at the edges of the quantum wire as a result of the quantum mechanical barrier penetration included within the TFDW approximation.

In Fig. 2, we have constructed a 'phase' diagram in the $\left(a k_{f}-V_{0} / E_{f}\right)$ plane which marks the boundary between localized and delocalized solutions of Eq. (四). To generate these curves, we have used the criterion $n_{0}\left( \pm \frac{a}{2}\right)=0.1 \bar{n}_{2 D}$. This condition is rather arbitrary, and a value somewhat greater than or less than $1 / 10$ could have been used to generate a similar set of curves. The solid and dashed curves correspond to average $2 \mathrm{D}$ densities of $\bar{n}_{2 D}=0.1$ and $\bar{n}_{2 D}=1.0$, respectively and span the range of densities of physical interest. The open circles represent the 'phase' boundary in the case of noninteracting electrons obtained by ignoring the electrostatic and exchange potentials in (5). In this limit, one can show from Eq. (5) that the scaled wavefunction $k_{f} \psi_{0}(x)$ as a function of the scaled length $x / a$ depends on the density only through the two parameters $a k_{f}$ and $V_{0} / E_{f}$. As a result, the phase boundary in the $\left(a k_{f}-V_{0} / E_{f}\right)$ plane is a universal curve which is valid for any density. It can be seen that this scaling property of the wavefunction does not apply once interactions are included. We have also indicated by the labeled points the values of the parameters corresponding to three of the density profiles in Fig. 1. These were obtained for a density of $\bar{n}_{2 D}=0.1$, so that the position of the points relative to the solid curve in Fig. 22 is relevant; point "(b)" is associated with a strongly modulated but extended density while point "(c)" is well into the localized region.

The parameter $a k_{f}$ can be used to distinguish two regimes of interest: the rapidly varying regime $a k_{f} \ll 1$ where the modulation period is small compared to the Fermi wavelength of the average density, and the slowly varying regime $a k_{f} \gg 1$. In the former limit, all of the curves approach an asymptote which is weakly dependent on density. This limit of strong quantum confinement can be understood simply in terms of the criterion that the potential barrier height, $V_{0}$, is large compared to the quantum mechanical zero point energy $\hbar^{2} / m a^{2}$. We thus conclude that the 'phase' boundary is given approximately by $V_{0} / E_{f} \sim\left(a k_{f}\right)^{-2}$, which is consistent with the numerical calculations. In this limit, the quantum kinetic energy is deciding the question of localization. In the opposite limit of a slowly varying potential $\left(a k_{f} \gg 1\right)$, the von Weizsäcker kinetic energy becomes negligible and we recover the Thomas-Fermi (TF) approximation. The experimental situations of interest are typically in this TF limit. The 'phase' boundary now approaches a straight line with zero slope in the noninteracting case (open circles) and finite slope in the interacting case (solid and dashed lines). The behavior of the noninteracting curve follows from the TF density, $n_{T F}(x)=\bar{n}_{2 D}+\frac{V_{0}}{\pi} \cos \left(\frac{2 \pi x}{a}\right)$, and our criterion for localization implies $V_{0} / E_{f} \simeq 1$, which is to be expected when the energy of the gas is exclusively kinetic. On the other hand, the behavior of the interacting 'phase' boundaries can be understood in electrostatic terms. The external potential $v_{\text {ext }}(x)$ can be viewed as arising from a modulation of the external positive background density about the average value $\bar{n}_{2 D}$ : the amplitude of this density modulation, $\Delta n_{\text {ext }}$, is related to $V_{0}$ by $V_{0}=\frac{2 \pi}{G} \Delta n_{\text {ext }}=a \Delta n_{\text {ext }}$. Since the electrons simply 
neutralize the positive background locally in the TF limit, the criterion for localization becomes $V_{0} \simeq a \bar{n}_{2 D}$, which implies $V_{0} / E_{f} \simeq \frac{1}{\pi k_{f}}\left(a k_{f}\right)$. Thus, the 'phase' boundary has a slope inversely proportional to the square root of the average density, consistent with the results in Fig. 2.

We may also consider general solutions of Eq. (4) which for the case of one-dimensional periodic modulation will take the form of Bloch-like states in the $x$ direction, $\psi_{n q_{x}}(x)=$ $e^{i q_{x} x} u_{n q_{x}}(x)$, where $u_{n q_{x}}(x)$ is a periodic function of $x$, multiplied by a plane wave factor, $e^{i q_{y} y}$, in the $y$ direction. Here, $q_{x}$ is restricted to the first Brioullin zone, $-\pi / a \leq q_{x} \leq \pi / a$, and $n$ is a band index. The $\mathbf{q}=0$ state in the lowest band is the ground state $\psi_{0}(x)$ considered previously. Although the significance of these general solutions is not immediately apparent, we shall see that they have some relevance to the calculation of the collective excitations in a modulated 2DEG, which similarly exhibit a Bloch-like structure. For the purpose of comparison with the plasmon bands to be calculated, we show in Fig. 3 the TFDW energy bands in the $x$ direction for the case of moderate modulation ("(b)" in Fig. 1).

\section{COLLECTIVE EXCITATIONS}

\section{A. Hydrodynamic Equations}

In order to determine the plasma modes in the modulated 2DEG, we adopt the TFDW hydrodynamic approach developed previously 22 . This is based on the usual continuity equation

$$
\frac{\partial n}{\partial t}+\nabla \cdot(n \mathbf{v})=0
$$

and the momentum equation

$$
n\left[\frac{\partial \mathbf{v}}{\partial t}+\mathbf{v} \cdot \nabla \mathbf{v}\right]=n \mathbf{F}-n \mathbf{v} \times \boldsymbol{\omega}_{c}
$$

where $\mathbf{F}=\mathbf{F}_{\text {int }}+\mathbf{F}_{\text {ext }}$ includes both the internal force acting on the electrons,

$$
\mathbf{F}_{\text {int }}(\mathbf{r}, t)=-\nabla\left[v_{\mathrm{eff}}(\mathbf{r}, t)-\frac{\lambda_{w}}{2} \frac{\nabla^{2} \psi(\mathbf{r}, t)}{\psi(\mathbf{r}, t)}\right],
$$

as well as any additional time-dependent external force, $\mathbf{F}_{\text {ext }}(\mathbf{r}, t)$. The potential term $v_{\text {eff }}$ in Eq. (13) contributes the expected force corresponding to the internal TF pressure and Coulomb-derived potentials, while the remaining term is associated with the von Weizsäcker kinetic energy. For completeness, we have also included in Eq. (12) the magnetic force due to an externally applied magnetic field, $\mathbf{B}$, which we shall take to be uniform and perpendicular to the 2DEG (i.e., $\mathbf{B}=B \hat{\mathbf{z}}$ ). This force is expressed in terms of the cyclotron frequency vector $\boldsymbol{\omega}_{c}=e \mathbf{B} / m^{*} c$. Although we defer an explicit treatment of magnetoplasma modes to a future paper, we shall develop the equations for this more general situation as very little additional effort is needed to do so.

Self-consistent solutions to our hydrodynamic equations are obtained by linearizing the density in small deviations from equilibrium, viz., $n \rightarrow n_{0}+\delta n$. If the time-dependent 
density is represented as $n=\psi^{2}$, the fluctuation of the von Weizsäcker wave function, $\delta \psi$, is related to the density fluctuation by $\delta n=2 \psi_{0} \delta \psi$. Retaining only first order quantities (the velocity $\mathbf{v}$ is itself first order), Eqs. (11) and (12) yield

$$
\frac{\partial \delta n}{\partial t}+\nabla \cdot\left(n_{0} \mathbf{v}\right)=0
$$

and

$$
\frac{\partial \mathbf{v}}{\partial t}=\delta \mathbf{F}-\mathbf{v} \times \boldsymbol{\omega}_{c}
$$

where the fluctuating force is given by

$$
\delta \mathbf{F}=-\nabla\left[\delta v_{\mathrm{eff}}-\frac{\lambda_{w}}{2 \psi_{0}} \nabla^{2} \delta \psi+\frac{\lambda_{w}}{2} \frac{\nabla^{2} \psi_{0}}{\psi_{0}^{2}} \delta \psi\right]+\delta \mathbf{F}^{\mathrm{ext}}
$$

with

$$
\delta v_{\mathrm{eff}}=4 C_{1} \psi_{0} \delta \psi-\frac{3}{2} C_{3} \delta \psi+\delta \phi
$$

The bracketed term in Eq. (16) will be denoted by $f$, so that $\delta \mathbf{F}^{\text {int }}=-\nabla f$.

For the purpose of determining the normal mode frequencies of the system and the associated mode densities, $\delta \mathbf{F}^{\text {ext }}$ can be set to zero. We shall later consider the response of the system to external fields in the calculation of the power absorption. Due to the translational invariance in the $y$-direction, all of the fluctuating variables $(\delta n, \delta \psi, f$ and $\mathbf{v})$ will have the form of a propagating wave $e^{i\left(q_{y} y-\omega t\right)}$ with $x$-dependent amplitudes. Making use of this dependence, Eqs. (14) and (15) can be expressed in the form

$$
-i \omega \delta n+i n_{0} q_{y} v_{y}+\frac{\partial}{\partial x}\left(n_{0} v_{x}\right)=0
$$

and

$$
\left(\omega^{2}-\omega_{c}^{2}\right) \mathbf{v}=i \omega \delta \mathbf{F}-\left(\boldsymbol{\omega}_{c} \times \delta \mathbf{F}\right)
$$

respectively. In these equations, only the $x$-dependent amplitudes are displayed. Recalling that $\delta \mathbf{F}=-\nabla f$, we have

$$
\boldsymbol{\omega}_{c} \times \delta \mathbf{F}=\left(i q_{y} \omega_{c} f\right) \hat{\mathbf{x}}-\left(\omega_{c} \frac{\partial f}{\partial x}\right) \hat{\mathbf{y}} .
$$

The use of Eq. (20) in Eq. (19), along with Eq. (18), then leads to

$$
\omega\left(\omega^{2}-\omega_{c}^{2}\right) \delta n=q_{y}^{2} \omega n_{0} f-\omega \frac{\partial}{\partial x}\left(n_{0} \frac{\partial f}{\partial x}\right)-\omega_{c} q_{y} \frac{\partial n_{0}}{\partial x} f .
$$

Eq. (21) can also be expressed in terms of the fluctuating wave function by using $n_{0}=\psi_{0}^{2}$ and $\delta n=2 \psi_{0} \delta \psi$. With this substitution, we find 


$$
\omega\left(\omega^{2}-\omega_{c}^{2}\right) \delta \psi=\frac{1}{2} q_{y}^{2} \omega \psi_{0} f-\omega \psi_{0}^{\prime} f^{\prime}-\frac{1}{2} \omega \psi_{0} f^{\prime \prime}-\omega_{c} q_{y} \psi_{0}^{\prime} f
$$

where derivatives with respect to $x$ are now denoted by primes. Noting that $\psi_{0} f^{\prime \prime}=\left(\psi_{0} f\right)^{\prime \prime}-$ $\psi_{0}^{\prime \prime} f-2 \psi_{0}^{\prime} f^{\prime}$, Eq. (22) yields,

$$
\begin{aligned}
\omega\left(\omega^{2}-\omega_{c}^{2}\right) \delta \psi & =\frac{1}{2} q_{y}^{2} \omega\left(\psi_{0} f\right)+\frac{1}{2} \omega \frac{\psi_{0}^{\prime \prime}}{\psi_{0}}\left(\psi_{0} f\right) \\
& -\frac{1}{2} \omega\left(\psi_{0} f\right)^{\prime \prime}-\omega_{c} q_{y} \frac{\psi_{0}^{\prime}}{\psi_{0}}\left(\psi_{0} f\right) .
\end{aligned}
$$

The advantage of this form is that the function $\left(\psi_{0} f\right)$ and its derivatives now appear on the right hand side, where

$$
\begin{aligned}
\psi_{0} f & =\psi_{0} \delta v_{\text {eff }}+\frac{\lambda_{w}}{2}\left(\frac{\psi_{0}^{\prime \prime}}{\psi_{0}}+q_{y}^{2}\right) \delta \psi-\frac{\lambda_{w}}{2} \delta \psi^{\prime \prime} \\
& \equiv \hat{M} \delta \psi+\hat{h} \delta \psi .
\end{aligned}
$$

The operator $\hat{M}$ is defined by $\hat{M} \delta \psi \equiv \psi_{0} \delta v_{\text {eff }}$ and

$$
\hat{h}=-\frac{\lambda_{w}}{2}\left(\frac{d^{2}}{d x^{2}}-q_{y}^{2}\right)+v_{\text {eff }}-\mu
$$

is just the Hamiltonian (for $q_{y}=0$ ) determining the ground state von Weizsäcker wave function $\psi_{0}(x)$. Substituting Eq. (24) into (23) yields

$$
\omega\left(\omega^{2}-\omega_{c}^{2}\right) \delta \psi=\omega \lambda_{w}^{-1} \hat{h}(\hat{h}+\hat{M}) \delta \psi-\omega_{c} q_{y} \frac{\psi_{0}^{\prime}}{\psi_{0}}(\hat{h}+\hat{M}) \delta \psi .
$$

In the limit of vanishing magnetic field, this reduces to

$$
\lambda_{w} \omega^{2} \delta \psi=\hat{h}(\hat{h}+\hat{M}) \delta \psi .
$$

Due to the periodicity induced by the modulating potential along the $x$-direction, the fluctuating part of the wave function will have the Bloch-like form

$$
\delta \psi=e^{i q_{x} x} \sum_{G} c_{G} \varphi_{G}
$$

where $c_{G}$ is a Fourier expansion coefficient and $\varphi_{G}=\frac{1}{\sqrt{a}} e^{i G x}$. These basis functions have been chosen to satisfy the orthonormality condition

$$
\int_{-a / 2}^{a / 2} d x \varphi_{G}^{*}(x) \varphi_{G^{\prime}}(x)=\delta_{G G^{\prime}}
$$

where $G=\frac{2 \pi n}{a}(n=0, \pm 1, \pm 2, \ldots)$ is a one-dimensional reciprocal lattice vector in the $x$-direction. Substituting the Fourier expansion (28) into (26), we obtain the equation

$$
\omega\left(\omega^{2}-\omega_{c}^{2}\right) c_{G}=-\omega_{c} q_{y} \sum_{G^{\prime} G^{\prime \prime}} A_{G G^{\prime}} \tilde{M}_{G^{\prime} G^{\prime \prime}} c_{G^{\prime \prime}}+\omega \sum_{G^{\prime} G^{\prime \prime}} B_{G G^{\prime}} \tilde{M}_{G^{\prime} G^{\prime \prime}} c_{G^{\prime \prime}},
$$


where the various matrices appearing in this equation are defined as

$$
\begin{aligned}
A_{G G^{\prime}} & =\frac{i}{\sqrt{a}}\left(G-G^{\prime}\right) \overline{\ln \psi_{0}}\left[G-G^{\prime}\right], \\
B_{G G^{\prime}} & \equiv \lambda_{w}^{-1} h_{G G^{\prime}} \\
& =\frac{1}{2}\left[\left(q_{x}+G\right)^{2}+q_{y}^{2}\right] \delta_{G G^{\prime}}+\frac{1}{\lambda_{w} \sqrt{a}} \overline{\left(v_{\mathrm{eff}}-\mu\right)}\left[G-G^{\prime}\right],
\end{aligned}
$$

and

$$
\tilde{M}_{G G^{\prime}}=M_{G G^{\prime}}+\lambda_{w} B_{G G^{\prime}} .
$$

In obtaining (31) we have used the identity $\frac{\psi_{0}^{\prime}}{\psi_{0}}=\frac{d}{d x}\left(\ln \psi_{0}\right)$, and denote the Fourier transform of a periodic function with an overline, e.g. $\psi_{0}(x)=\sum_{G} \overline{\psi_{0}}[G] \varphi_{G}$.

The remaining matrix $M_{G G^{\prime}}$ in (33) consists of several terms. From Eq. (24), we have

$$
\begin{aligned}
\hat{M} \delta \psi & =\psi_{0} \delta v_{\text {eff }} \\
& =4 C_{1} \psi_{0}^{2} \delta \psi-\frac{3}{2} C_{3} \psi_{0} \delta \psi+\psi_{0} \delta \phi .
\end{aligned}
$$

The last term in (34) involves the $x$-dependent part of the total electric potential fluctuation, $\delta \phi$. In terms of the density fluctuation $\delta n$, we have

$$
\begin{aligned}
\delta \phi(\mathbf{r}) & =\int d \mathbf{r}^{\prime} \frac{\delta n\left(\mathbf{r}^{\prime}\right)}{\left|\mathbf{r}-\mathbf{r}^{\prime}\right|} \\
& =\int d \mathbf{r}^{\prime} \frac{2 \psi_{0}\left(x^{\prime}\right) e^{i\left(q_{x} x^{\prime}+q_{y} y^{\prime}\right)} \sum_{G^{\prime}} c_{G^{\prime}} \varphi_{G^{\prime}}\left(x^{\prime}\right)}{\left|\mathbf{r}-\mathbf{r}^{\prime}\right|} \\
& =e^{i\left(q_{x} x+q_{y} y\right)} \frac{4 \pi}{a} \sum_{G G^{\prime}} \frac{e^{i G x}}{\sqrt{\left(q_{x}+G\right)^{2}+q_{y}^{2}}} \overline{\psi_{0}}\left[G-G^{\prime}\right] c_{G^{\prime}},
\end{aligned}
$$

where we have noted that the 2D Fourier transform of $r^{-1}$ is $(2 \pi / q)$. It is clear from this expression that $\delta \phi(\mathbf{r})$ is proportional to $e^{i q_{y} y}$ and that the $x$-dependent part has the expected Bloch-like form. With this result, (34) yields

$$
M_{G G^{\prime}}=M_{G G^{\prime}}^{K}+M_{G G^{\prime}}^{X}+M_{G G^{\prime}}^{H},
$$

where

$$
\begin{aligned}
& M_{G G^{\prime}}^{K}=\frac{4 C_{1}}{\sqrt{a}} \overline{\psi_{0}^{2}}\left[G-G^{\prime}\right], \\
& M_{G G^{\prime}}^{X}=-\frac{3 C_{3}}{2 \sqrt{a}} \overline{\psi_{0}}\left[G-G^{\prime}\right], \\
& M_{G G^{\prime}}^{H}=\frac{4 \pi}{a} \sum_{G^{\prime \prime}} \frac{\overline{\psi_{0}}\left[G-G^{\prime \prime}\right] \overline{\psi_{0}}\left[G^{\prime \prime}-G^{\prime}\right]}{\sqrt{\left(q_{x}+G^{\prime \prime}\right)^{2}+q_{y}^{2}}} .
\end{aligned}
$$


The superscripts $K, X$ and $H$ refer respectively to the kinetic, exchange and Hartree terms in $\psi_{0} \delta v_{\text {eff }}$. It is clear that the kinetic and exchange matrices depend on $G-G^{\prime}$, but the Hartree matrix, which is associated with a nonlocal operator in position space, has the property $M_{G+G_{0}, G^{\prime}+G_{0}}^{H}\left(q_{x}\right)=M_{G G^{\prime}}^{H}\left(q_{x}+G_{0}\right)$.

Eq. (30) is a nonlinear eigenvalue problem which must be solved numerically for the eigenvalue $\omega$ and eigenvector $\vec{c}$. In principle, the dimension of the eigenvalue problem is infinite, and for practical purposes, a truncation of the expansion to some finite number of $G$ vectors is required. However, one can always check that the results for the modes of interest have converged by systematically increasing the number of $G$ vectors. Once the eigenvalues $\omega$ have been determined, the corresponding eigenvectors $\vec{c}$ can be substituted into Eq. (28) to determine the mode densities.

To close this section, we note some general consequences of the equations we have obtained. In the $q_{y} \rightarrow 0$ limit, (30) reduces to

$$
\left(\omega^{2}-\omega_{c}^{2}\right) c_{G}=\sum_{G^{\prime} G^{\prime \prime}} B_{G G^{\prime}} \tilde{M}_{G^{\prime} G^{\prime \prime}} c_{G^{\prime \prime}}
$$

and the magnetic field appears explicitly only on the left hand side of the equation. This implies that the magnetoplasma frequencies have the property

$$
\omega_{n}^{2}\left(q_{x}, q_{y}=0 ; B\right)=\omega_{n}^{2}\left(q_{x}, q_{y}=0 ; B=0\right)+\omega_{c}^{2},
$$

that is, a simple cyclotron shift of the zero-field frequencies, $\omega_{n}\left(q_{x}, q_{y}=0 ; B=0\right)$. In addition, the mode densities in this limit have exactly the same spatial distribution as the corresponding $B=0$ mode densities.

Another general property can be deduced from Eq. (27) by making use of the Blochstate basis, $\psi_{n q_{x}}(x)$, introduced at the end of Sec. II. These states are eigenstates of $\hat{h}$ with eigenvalues $\varepsilon_{n}(\mathbf{q})=E_{n}\left(q_{x}\right)+\frac{1}{2} \lambda_{w} q_{y}^{2}-\mu$, where $E_{n}\left(q_{x}\right)$ are the $1 \mathrm{D}$ band energies and the chemical potential $\mu$ is equal to $E_{0}(0)$, the $\mathbf{q}=0$ energy of the lowest band $(n=0)$. Expanding $\delta \psi$ as

$$
\delta \psi=\sum_{n} d_{n} \psi_{n q_{x}}
$$

and substituting this expansion into Eq. (27), we obtain the eigenvalue problem in the alternate form

$$
\lambda_{w} \omega^{2} d_{n}=\varepsilon_{n}(\mathbf{q}) \sum_{n^{\prime}}\left[\varepsilon_{n}(\mathbf{q}) \delta_{n n^{\prime}}+M_{n n^{\prime}}\right] d_{n^{\prime}}
$$

By its definition, $\varepsilon_{0}(0)=0$ for the lowest band, and as a result, Eq. (43) will have a nontrivial solution at $\mathbf{q}=0$ with frequency $\omega=0$. In other words, the lowest plasmon band disperses from zero at $\mathbf{q}=0$ and, according to Eq. (41), this implies that there will be a magnetoplasmon branch which disperses from $\omega_{c}$.

\section{B. Power Absorption}

The main method for studying the collective modes in these systems is by means of FIR absorption experiments 192428 . To make contact with these experiments, we consider in this 
section the calculation of the power absorption which, in addition to the mode frequencies, contains information about the oscillator strengths of the observed excitations.

The instantaneous power absorption is given by

$$
P(t)=\int d \mathbf{r} \mathbf{j}^{\text {ind }}(\mathbf{r}, t) \cdot \mathbf{E}^{\mathrm{ext}}(\mathbf{r}, t)
$$

where $\mathbf{j}^{\text {ind }}(\mathbf{r}, t)$ is the current induced by an external electromagnetic field. For a uniform radiation field polarized in the $x$-direction and incident normally on the sample, the external field is spatially uniform and is given by $\mathbf{E}^{\operatorname{ext}}(\mathbf{r}, t)=\frac{1}{2} E_{0}\left(e^{-i \omega t}+e^{i \omega t}\right) \hat{\mathbf{x}}$. The physically relevant quantity is the time-averaged power absorption which is given by

$$
\langle P\rangle_{t}=\frac{1}{2} E_{0} \int d \mathbf{r} \operatorname{Re} j_{x}^{\text {ind }}(\mathbf{r}, \omega)
$$

Since the induced current $j_{x}^{\text {ind }}$ for the situation of interest is a periodic function of $x$ and independent of $y$, the time-averaged power absorption per unit area is given by

$$
\frac{\langle P\rangle_{t}}{A}=\frac{1}{2 \sqrt{a}} E_{0} \operatorname{Re} \overline{j_{x}^{\text {ind }}}[G=0, \omega]
$$

where

$$
\overline{j_{x}^{\text {ind }}}[G, \omega]=\frac{1}{\sqrt{a}} \int_{-a / 2}^{a / 2} d x e^{-i G x} j_{x}^{\text {ind }}(x, \omega)
$$

is the Fourier coefficient of the induced current.

The current density is determined by $j_{x}^{\text {ind }}(x, \omega)=-n_{0}(x) v_{x}(x, \omega)$ where the velocity is the solution of

$$
\frac{\partial \mathbf{v}}{\partial t}=-\gamma \mathbf{v}+\delta \mathbf{F}-\mathbf{v} \times \boldsymbol{\omega}_{c}
$$

We have included in this equation a phenomenological relaxation rate, $\gamma$, which accounts for momentum nonconserving scattering processes of the electrons. Because of the relaxation rate, the frequency $\omega$ appearing in Eq. (19) is now replaced by $\tilde{\omega}=\omega+i \gamma$. Noting that $\delta \mathbf{F}$ now includes the additional term $\delta F_{x}^{\text {ext }}=-E_{0}$, we obtain the following expression for the current density at $\mathbf{q}=0$,

$$
j_{x}^{\text {ind }}(x, \omega)=-n_{0}(x) v_{x}(x, \omega)=\frac{i \tilde{\omega}}{\tilde{\omega}^{2}-\omega_{c}^{2}} n_{0}\left(\frac{\partial f}{\partial x}+E_{0}\right) .
$$

Taking the $\mathbf{q}=0$ limit of the continuity equation in (18), along with Eq. (49), we find

$$
\omega\left(\tilde{\omega}^{2}-\omega_{c}^{2}\right) \delta n=-\tilde{\omega} \frac{\partial}{\partial x}\left(n_{0} \frac{\partial f}{\partial x}\right)-\tilde{\omega} E_{0} \frac{\partial n_{0}}{\partial x} .
$$

Thus, the net effect of including the external driving field $\mathbf{E}^{\text {ext }}$ is to convert the eigenvalue problem in Eq. (30), into the set of inhomogeneous equations

$$
\omega\left(\tilde{\omega}^{2}-\omega_{c}^{2}\right) c_{G}-\tilde{\omega} \sum_{G^{\prime} G^{\prime \prime}} B_{G G^{\prime}} \tilde{M}_{G^{\prime} G^{\prime \prime}} c_{G^{\prime \prime}}=-i \tilde{\omega} G \overline{\psi_{0}}[G] E_{0} .
$$


Substituting Eq. (49) into Eq. (47), we obtain

$$
\overline{j_{x}^{\text {ind }}}[G=0, \omega]=\frac{-2 \tilde{\omega}}{\sqrt{a}\left(\tilde{\omega}^{2}-\omega_{c}^{2}\right)} \sum_{G} G \overline{\psi_{0}}[G] f_{G}+\frac{i \tilde{\omega} E_{0}}{\tilde{\omega}^{2}-\omega_{c}^{2}} \overline{n_{0}}[G=0],
$$

where, in deriving this result, we have used the fact that $\left(n_{0} f\right)$ is periodic, $n_{0}=\psi_{0}^{2}$, and $\overline{\psi_{0}}[-G]=\overline{\psi_{0}}[G]$. The quantity $f_{G}$ is the Fourier coefficient of $\left(\psi_{0} f\right)$ defined in Eq. (24), and is related to $c_{G}$ by the equation

$$
f_{G}=\sum_{G^{\prime}} \tilde{M}_{G G^{\prime}} c_{G^{\prime}}
$$

The substitution of (52) into (46) provides our final expression for the power absorption. Since $\overline{n_{0}}[G=0]=\sqrt{a} \bar{n}_{2 D}$, the last term in Eq. (52) contributes an absorption peak at the cyclotron frequency, $\omega_{c}$, as found for a uniform 2DEG. This peak shifts to $\omega=0$ for $B=0$, and represents the expected Drude-like absorption associated with the resistive losses in the 2DEG. The other term on the right hand side of Eq. (52) accounts for the density inhomogeneity and has the effect of reducing the amount of Drude absorption.

The cancellation of the Drude peak can be seen more clearly by writing the power absorption in an alternate form. Starting with the continuity equation (18) with $\mathbf{q}=0$

$$
i \omega \delta n+\frac{\partial}{\partial x} j_{x}^{\text {ind }}=0
$$

multiplying by $x$ and integrating over a unit cell, we find

$$
\overline{j_{x}^{\text {ind }}}[G=0, \omega]=\frac{i \omega}{\sqrt{a}} \int_{-a / 2}^{a / 2} x \delta n(x) d x+\sqrt{a} j^{\text {ind }}(a / 2) .
$$

The substitution of this expression into Eq. (46) then yields

$$
\frac{\langle P\rangle_{t}}{A}=-\frac{1}{2} \omega E_{0} \operatorname{Im} \frac{1}{a} \int_{-a / 2}^{a / 2} x \delta n(x, \omega) d x+\frac{1}{2} E_{0} \operatorname{Re} j_{x}^{\text {ind }}(x=a / 2, \omega) .
$$

In the localized limit, the boundary current $j_{x}^{\text {ind }}(x=a / 2, \omega)$ vanishes and the power absorption is then determined by the induced dipole moment of the charge density fluctuation. In this case, there is no absorption at the cyclotron frequency, $\omega_{c}$, despite the appearance of the resonant denominators in Eq. (52). In the opposite limit of a weakly modulated system, the induced dipole moment will be small and the power absorption will be dominated by the second term in (55). We then recover the Drude absorption discussed previously.

\section{THE 2D TO 1D CROSSOVER}

In this section, we study the plasma modes of a 2DEG subjected to the modulating potential given in Eq. (6). Our main interest is in the evolution of these modes as a function of the strength of the modulation. The crossover from 2D to $1 \mathrm{D}$ behaviour will occur in the vicinity of the 'phase' boundary illustrated in Fig. 1. Once the modulation is sufficiently strong, the electron layer separates into an array of quantum wires and we can expect very 
different behaviour from the original 2D situation. Although the theory in Sec. III was developed with the inclusion of a magnetic field, we shall restrict our investigation to the zero field limit, in which case Eq. (30) reduces to

$$
\omega^{2} c_{G}=\sum_{G^{\prime}, G^{\prime \prime}} B_{G G^{\prime}} \tilde{M}_{G^{\prime} G^{\prime \prime}} c_{G^{\prime \prime}}
$$

All the mode frequencies presented in this section are based on an analysis of this equation.

\section{A. The Uniform 2DEG}

It is useful for the purpose of orientation to begin with the homogeneous 2DEG $\left(v_{\text {ext }}=0\right)$ as treated in the TFDW approximation. The interaction matrices in Eqs. (37)-(39), as well as the $B$-matrix in (32), are diagonal in this case and we readily obtain from (57) the plasmon dispersion relation

$$
\begin{aligned}
\omega_{0}^{2}(q) & =2 \pi \bar{n}_{2 D} q+\left(2 C_{1} \bar{n}_{2 D}-\frac{3}{4} C_{3} \sqrt{\bar{n}_{2 D}}\right) q^{2}+\frac{\lambda_{w}}{4} q^{4} \\
& =k_{f}^{2} q+\left(\frac{1}{2} k_{f}^{2}-\frac{1}{\pi} k_{f}\right) q^{2}+\frac{\lambda_{w}}{4} q^{4} .
\end{aligned}
$$

At long wavelengths, this gives the expected 2D plasmon frequency $\omega_{2 D}=\sqrt{2 \pi \bar{n}_{2 D} q}$ which has the characteristic $\sqrt{q}$ dependence. At shorter wavelengths, the effects of the TF $\left(C_{1}\right)$, exchange $\left(C_{3}\right)$ and von Weizsäcker $\left(\lambda_{w}\right)$ energies become of increasing importance. It is interesting to note that the exchange interaction gives a negative $q^{2}$ coefficient which counteracts the positive dispersion coming from the TF kinetic energy. As a result, the $q^{2}$ coefficient goes to zero at a density having $k_{f}=2 / \pi$.

\section{B. Weak Modulation}

We now consider a weak modulating potential corresponding to the phase-point "(a)" in Fig. 2, with coordinates $\left(a k_{f}=31.71, V_{0} / E_{f}=6.4\right)$. In Fig. 田 we illustrate the $q_{x}$-dispersion for $q_{y}=0$, as determined from Eq. (57). Also shown for comparison as the solid line is the uniform 2DEG dispersion relation given by Eq. (58). The similarity of the dispersion curves for the two cases is notable. The only obvious difference is the appearance of a gap in the plasmon dispersion at the zone boundary, $q_{x}=\pi / a$, which is associated with the development of plasmon bands. This problem was treated theoretically by Krasheninnikov and Chaplik 5 using degenerate perturbation theory. They found that the size of the gaps induced by the density modulation is given by $\Delta \sim\left(\left|N_{n}\right| / \bar{n}_{2 D}\right) \omega_{n}$, where $\omega_{n}$ is the unperturbed plasma frequency for wavevector $q_{x} \equiv q_{n}=n \pi / a$ and $N_{n}$ is the $n$th coefficient in the Fourier expansion of the $2 \mathrm{D}$ electron density $\left(N_{n}=\overline{n_{0}}[2 \pi n / a] / \sqrt{a}\right.$ in our notation; odd values of $n$ give rise to zone boundary (ZB) gaps, while even values give rise to zone center (ZC) gaps). Since their analysis is based on a different formulation of the problem and includes only the Hartree interaction, we present for completeness a perturbation theory calculation of the gaps within the TFDW hydrodynamics in Appendix A. We find that the gaps are determined by the equation 


$$
\omega_{ \pm}^{2}=\omega_{0}^{2}\left(q_{n}\right) \pm\left(\omega_{0}^{2}\left(q_{n}\right)-\frac{3}{8} C_{3} \sqrt{\bar{n}_{2 D}} q_{n}^{2}+\frac{3}{4} \lambda_{w} q_{n}^{4}\right)\left|\frac{\overline{n_{0}}[2 \pi n / a]}{\overline{n_{0}}[0]}\right|,
$$

which is consistent with the result of Krasheninnikov and Chaplik in the long wavelength limit where the Hartree interaction is dominant. In Fig. 15 of Appendix A, we show some of the Fourier coefficients $\overline{n_{0}}[2 \pi n / a]$ as a function of $V_{0} / E_{f}$. The $\overline{n_{0}}[2 \pi / a]$ coefficient is linear in $V_{0}$ whereas the higher Fourier components are at least of order $V_{0}^{2}$ and are therefore much smaller in the weakly modulated regime. This accounts for the relatively small magnitude of the gaps in Fig. 1 as compared to the lowest zone boundary gap.

In Fig. 5, we show the plasmon dispersion in the $y$ direction (i.e., perpendicular to the modulation direction) for modes at the zone center $\left(q_{x}=0\right.$, solid circles $)$ and at the zone boundary $\left(q_{x}=\pi / a\right.$, open circles). Specific modes of interest that will be referred to in the text are indicated by encircled numbers. Of the $q_{x}=0$ modes, there is one which starts at zero frequency (mode "1"), as explained at the end of Sec. IIIA. To determine the small- $q_{y}$ behaviour analytically, we make use of the long-wavelength form of $\tilde{M}_{G G^{\prime}}$ derived in Appendix B. It is shown there that the 2D plasmon dispersion for $q_{x}=0$ with $q_{y} \rightarrow 0$ is given by $\omega^{2} \simeq 2 \pi \bar{n}_{2 D} q_{y}$, the usual plasma frequency of a uniform $2 \mathrm{DEG}$. This is a general result independent of the degree of modulation of the 2DEG. In other words, the modulation of the 2DEG has no effect on the long-wavelength dispersion of plasmons in this direction. However, as discussed in more detail in Sec. IVC, this is not the case for plasmons propagating in the $x$ direction.

There are several other notable features in Fig. 5. The first concerns the modes at $q_{x}=\pi / a$ which behave as $\delta n(x+a)=-\delta n(x)$ and have a periodicity of $2 a$. The two lowest modes (the "2-8" and "3-7" branches) indicated by open circles in Fig. 5 cross close to $q_{y}=1$. This implies that the gap at the zone boundary first decreases to zero as a function of $q_{y}$, and then increases again. Such a crossing is possible since the two modes in question have different symmetries. To illustrate this more clearly, we show the induced charge density fluctuations for various modes in Fig. 6. The left panel of this figure corresponds to $q_{y}=0$ while the right panel is for $q_{y}=3 \pi / a$. The sequence from top to bottom illustrates the five lowest modes, starting with the $q_{x}=0$ mode in the top panel, followed by the two $q_{x}=\pi / a$ modes in the middle panel and finally the next pair of $q_{x}=0$ modes in the bottom panel. The right panel of Fig. [ shows how these mode densities change when $q_{y}$ is increased to $3 \pi / a$. It is the pair of $q_{x}=\pi / a$ modes in the middle panel which exhibits the frequency crossing in Fig. 5. For $q_{y}$ values up to the crossing point, the lower frequency mode (the "2-8" branch) is symmetric with respect to reflections about $x=0$, while the next highest mode (the "3-7" branch) is antisymmetric. The ordering in frequency of these two modes is then reversed at the crossing point, with the odd-parity mode lying lower. Similar behaviour was found for the model densities with weak modulation considered in Ref. [ 8], but for strong modulation an anticrossing behaviour was observed. As we shall see, the crossing behaviour we find persists for even stronger modulations (see Fig. 9).

A further examination of Fig. 5 shows the odd-parity mode at $q_{x}=\pi / a$ approaching the lowest-lying $q_{x}=0$ mode with increasing $q_{y}$ (points " 6 " and " 7 "). The reason for this can be explained by comparing the mode density ("6") in the top-left panel of Fig. 6 with the odd-parity density ("7") in the middle-right panel. Both mode densities are seen to be localized at the cell boundaries where the equilibrium density is lowest, and both have a very similar spatial profile in this region. The near degeneracy of these modes for large $q_{y}$ 
indicates that there is a weak interaction between the density fluctuations localized at adjacent cell boundaries and that as a result, these modes effectively propagate independently of each other. This behaviour is analogous to the situation in a metallic slab where the symmetric and antisymmetric surface plasmons become degenerate at large wave vectors. The localization of the mode density at the cell boundaries increases with increasing $q_{y}$, implying that the modes are channeled in their propagation along the low density part of the gas.

We also see a similar convergence of the other $q_{x}=\pi / a$ mode with the next higher $q_{x}=0$ mode at large $q_{y}$. The explanation for this is the same as given above. The density fluctuation "8" shown by the solid line in the right-middle panel of Fig. 6 is the even-parity version of the odd-parity density fluctuation "9" shown by the dashed curve in the lowerright panel. A similar pairing of $q_{x}=0$ and $q_{x}=\pi / a$ modes would also be expected for the higher lying modes in Fig. 5. This behaviour is in fact more evident when the modulation amplitude of the equilibrium density increases (see Figs. 9 and 12). We should emphasize that the convergence of pairs of $q_{x}=0$ and $q_{x}=\pi / a$ mode frequencies implies that these modes exhibit a very weak dispersion with respect to $q_{x}$. The absence of an effect of the $e^{i q_{x} x}$ phase modulation from one unit cell to the next reflects the lack of interaction between adjacent density fluctuations.

Returning to Fig. 4 , we see that the gaps at the zone center are unobservably small for this case of weak modulation. However, each of the higher lying modes at $q_{x}=0$ corresponds to two distinct modes. This becomes apparent for the first excited $q_{x}=0$ modes in Fig. 5 (starting at the point labeled " 4 " and "5") where it is seen that the frequencies separate with increasing $q_{y}$. The lower-left panel in Fig. 6 shows the mode densities for this pair of modes at $q_{y}=0$, and the lower-right panel shows the densities at $q_{y}=3 \pi / a$. One mode has even parity with respect to the center of the cell, and the other has odd parity. The latter mode eventually evolves with increasing modulation into the lowest odd-parity mode of an isolated wire. Since this mode has a finite dipole moment, it will couple to an external radiation field and will contribute to the power absorption as indicated by Eq. (56). To illustrate this, we show in Fig. 7 the calculated power absorption for different modulations of the 2DEG. The lowest curve labeled "(a)" corresponds to the case of weak modulation being considered here and shows a small peak at the frequency of the first excited $q_{x}=0$ mode " 4 ". This is essentially a bulk $2 \mathrm{D}$ plasmon at a wavevector $q=2 \pi / a$. In principle, other

$q_{x}=0$ odd-parity modes should be observable, but their oscillator strengths are too small to show up in the power absorption. In fact, the dominant feature in the power absorption of curve "(a)" is the strong Drude peak at $\omega=0$ which is to be expected since the system is only weakly perturbed from a homogeneous 2DEG. We shall return to a more systematic discussion of the power absorption later.

\section{Moderate Modulation}

As the modulation is increased, the equilibrium density profile becomes more localized (see the curve labeled by "(b)" in Fig. 1). The effect of this increased modulation on the wavevector dispersion along the $x$-direction is illustrated in Fig. 8. A comparison of this figure with Fig. 14 reveals several notable changes, the most dramatic being the increase in the magnitude of the gaps at both the zone center and zone boundary. As we have already 
discussed in Sec. IVB, the size of the gap is related to the magnitude of the density Fourier coefficients, so this result is to be expected. We also notice that the $q_{x}$-dispersion of the lowest branch is much flatter than for the uniform gas. The explanation for this behaviour is once again found in Appendix B where we show that the long-wavelength frequency of plasmons propagating in the $x$ direction is given by $\omega^{2}\left(q_{x}, q_{y}=0\right)=2 \pi \bar{n}_{2 D}\left(q_{x} / m_{x}\right)$, where $m_{x}$ is the effective band mass at the zone center. Since $m_{x}$ increases with $V_{0}$, the dispersion of the plasmon becomes weaker with increasing modulation.

In Fig. 9 we present the $q_{y}$-dispersion analogous to that shown in Fig. 5 for the case of weak modulation. As expected, the characteristic $\sqrt{q_{y}}$ behaviour is seen to persist (the "1-6" branch), but now the $2 \mathrm{D}$ plasmon crosses the lowest $q_{x}=\pi / a$ mode (the " $2-8$ " branch). At these crossing points, the dispersion of the plasmon band as a function of $q_{x}$ is weak since the frequencies at $q_{x}=0$ and $q_{x}=\pi / a$ are coincidentally equal. As for the case of weak modulation, we also see the lowest pair of $q_{x}=\pi / a$ modes (branches "2-8" and "3-7") crossing, implying that the lowest zone boundary gap closes at some finite value of $q_{y}$. This again is possible due to the different parities of these modes (" 2 " and " 3 ") in the middle panel of Fig. 10. The same behaviour is now seen for the next pair of $q_{x}=\pi / a$ modes which also have opposite parity. The parity of the modes is of course preserved as a function of $q_{y}$, but this is not the case as a function of $q_{x}$. The mode density " 1 " at $q_{y}=0$ in Fig. 10 evolves continuously into the mode density " 2 " as $q_{x}$ is increased from 0 to $\pi / a$, and the parity of the mode remains unchanged. On the other hand, at $q_{y}=3 \pi / a$, the mode density " 6 " evolves continuously into "7", and thus a change in parity is observed as the mode disperses with respect to $q_{x}$. This change in behaviour is associated with the crossing of the pair of $q_{x}=\pi / a$ branches " $2-8$ " and " $3-7$ ".

We again see in Fig. 9 the merging of zone boundary and zone center mode frequencies at large $q_{y}$ values. This behaviour is even clearer than in the case of weak modulation and the explanation is the same. The mode densities of the lowest two modes at $q_{y}=3 \pi / a$ are labeled "6" and "7" in Fig. 10; the density profiles of these two modes have a very similar shape near the cell boundaries where the equilibrium density is smallest, and only differ in the relative sign of the density fluctuation from one cell to the next. The weak interaction between the density fluctuations localized at adjacent cell boundaries accounts for the near degeneracy of these modes. The same can be said of the pair of density fluctuations labeled "8" and "9".

The nature of the mode densities at the lowest zone center gap is shown in the lower-left panel of Fig. 10. The mode labeled " 4 " is an odd-parity mode and contributes a peak to the power absorption near $\omega \simeq 0.25$ a.u. as shown by the curve labeled "(b)" in Fig. 7. It is this mode which eventually evolves into the center of mass mode in the limit of isolated $1 \mathrm{D}$ wires. There is an additional weak peak at $\omega \simeq 0.5$ a.u. corresponding to the next odd-parity mode at the zone center. It is clear that increasing the modulation has led to a decrease in the amplitude of the Drude peak at $\omega=0$ and a transfer of oscillator strength to the other zone center modes.

\section{Strong Modulation}

The system is in the strong modulation regime when the phase point lies above the solid curve in Fig. 2. In particular, the phase point “(c)" corresponds to an equilibrium density 
which is made up of an array of isolated quantum wires. Since there is no appreciable density overlap between adjacent unit cells, we expect a relatively flat $q_{x}$-dispersion for the lower plasmon bands. In Fig. 11, we show the $q_{x}$-dispersion for this case of strong modulation. Notice that the lowest plasmon branch has been pushed down to $\omega \approx 0$ (because of the large effective band mass $m_{x}$ ) and is dispersionless. Somewhat surprisingly, the next higher branch exhibits a dispersion which is larger than the next three branches lying between $\omega=0.5$ and $\omega=0.9$ a.u. This would not be the expected behaviour in a tight-binding model of electronic band structure in which the dispersion of the bands increases with increasing energy (see Fig. 31). This conventional band structure effect is beginning to be evident at the top of Fig. 11. As we shall see, the anomalous dispersion of the first excited band is due to inter-wire Coulomb interactions.

The dispersion of the plasmons in the $q_{y}$-direction is shown in Fig. 12. In contrast to the weak and moderately modulated cases, there are now two gapless modes present in the spectrum 37. The long-wavelength $\sqrt{q_{y}}$ character of the $q_{x}=02 \mathrm{D}$ plasmon " 1 " is still clearly apparent but there is a second mode " 2 " with $q_{x}=\pi / a$ which has a linear dispersion 38 . This branch originates from the "2-8" branch in Fig. 9 and in fact corresponds to a 1D plasmon propagating along each wire, but with a density fluctuation which changes sign from one wire to the next. The two lowest curves in Fig. 12 define the plasmon band for an array of quantum wires and is qualitatively similar to the results found previously for a quantum wire superlattice based on the RPA 6 , 1 or hydrodynamic model $\mathbf{B}$.

The next mode " 4 " in Fig. 12 is the center of mass (CM) mode for a 1D wire and the "4-9" and "3-7" branches define the CM plasmon band. This band is qualitatively similar to that found by Eliasson et al., Ref. [ 8]. The density fluctuation at $q_{x}=0$ and $q_{y}=0$ is shown by "4" in Fig. 13 and, to an excellent approximation, is simply the derivative of the equilibrium density profile shown by curve "(c)" in Fig. 1. This is the expected behaviour of the 'sloshing' CM mode for parabolic confinement 39 - As one disperses along $q_{x}$ to " 3 " at $q_{x}=\pi / a$, or along $q_{y}$ to " 9 " at $q_{y}=3 \pi / a$, one sees that the density fluctuations at all positions are essentially the same. Thus, this mode is an intra-wire mode with very little interaction between the wires.

Another interesting feature in Fig. 12 is the way in which the plasmon band merges with the CM band for large $q_{y}$. This is reminiscent of the mode dispersions found in 3D parabolic well 32 where the surface plasmon merges with the CM mode at higher wavevectors. The reason for the merging is the same as in the weak and moderate modulation cases; the density fluctuations shown by "7" and "8" in Fig. 13 are symmetric and antisymmetric versions of each other with very similar density profiles at the edge of the wires. At this large $q_{y}$ value, the density fluctuations on either side of the wire interact with each other only weakly, as do the fluctuations on different wires.

We turn next to the explanation of the finite bandwidth of the "3-4" branch in Fig. 11 . The origin of the dispersion is the dipole-dipole interaction between the wires. We consider the limiting case where the wire width $W$ is small compared to the inter-wire separation $a$. For $q_{y}=0$, the electric field experienced by the $n$-th wire in the dipole approximation is

$$
E_{n}=\frac{2}{a^{2}} \sum_{s}^{\prime} \frac{p_{n+s}}{s^{2}}
$$

where $p_{n}$ is the dipole moment per unit length and the sum over $s$ excludes the $s=0$ term. 
We now suppose that the dipole moment has a plane wave modulation in the $x$-direction: $p_{n}=p_{0} e^{i q_{x} n a}$. In this case, the electric field is given by

$$
E_{n}=\frac{2 p_{0}}{a^{2}} \mathrm{e}^{i q_{x} n a} \sum_{s} \frac{\mathrm{e}^{i q_{x} s a}}{s^{2}} .
$$

The quantities $p_{n}$ and $E_{n}$ are connected through the relation $p_{n}=\alpha(\omega) E_{n}$ where $\alpha(\omega)$ is the dipole polarizability of the wire. Since the confining potential for the wire is roughly parabolic, the polarizability is given by $\alpha(\omega)=\lambda /\left(\omega_{0}^{2}-\omega^{2}\right)$, where $\lambda=a \bar{n}_{2 D}$ is the line density of the wire and $\omega_{0} \simeq \sqrt{2 \pi^{2} V_{0} / a^{2}}$ is the frequency of the harmonic potential. Using this result in Eq. (61), we find the following dispersion relation for the CM band:

$$
\omega^{2}=\omega_{0}^{2}-\frac{4 \lambda}{a^{2}} \sum_{s=1}^{\infty} \frac{\cos \left(q_{x} a s\right)}{s^{2}} .
$$

This result is entirely consistent with the observed $q_{x}$ dispersion for the " $3-4$ " branch shown in Fig. 11.

Finally, we discuss the FIR power absorption in Fig. 7. Each successive curve corresponds to a modulation increase of $\Delta V_{0}=1.0$ a.u., starting at $V_{0}=2.0$ a.u. The curves labeled "(a)" and "(b)" were discussed earlier in the context of weak and moderate modulation, respectively. We note that an increase in the strength of the modulation transfers oscillator strength from the Drude peak to the higher $q_{x}=0$ modes having a nonzero dipole moment. The two peaks which grow in intensity between curves "(a)" and "(b)" evolve from the $q=2 \pi / a$ and $q=4 \pi / a$ 2D bulk plasmons. Both peaks are red-shifted with increasing modulation with respect to their positions in the uniform gas limit. Near the curve labeled "(b)", this trend is reversed and the peak frequencies begin to increase with further increase in modulation. At the same time, the oscillator strength of the lower peak continues to increase, while that of the higher frequency peak begins to decrease. Beyond this point we also see a rapid reduction in the strength of the Drude peak at $\omega=0$.

The change in behaviour of the peak positions and intensities near the curve "(b)" occurs as the 2D-1D phase boundary in Fig. 2 is being crossed. Thus, a signature of the $2 \mathrm{D} \rightarrow$ $1 \mathrm{D}$ transition is the observation of a minimum in the fundamental resonance frequency as a function of modulation, and the disappearance of the Drude peak. Beyond this minimum. the fundamental resonance grows in intensity as it evolves into the CM (Kohn) mode 3941 for parabolic confinement. For large modulations, the quantum wires are well-separated and sit in an effectively harmonic potential with frequency $\omega_{0} \simeq \sqrt{2 \pi^{2} V_{0} / a^{2}}$, which explains the increasing trend observed in Fig. 1 . This also explains why the fundamental resonance eventually exhausts the total FIR oscillator strength.

As an overview of our results, we plot in Fig. 14 the zone boundary and zone center frequencies at $q_{y}=0$ as a function of the strength of the modulating potential. Only the lowest ZB modes exhibit a linear separation of the frequency with $V_{0}$ and is in accord with our perturbation theory results. The lower branch is seen to tend to zero as $V_{0}$ increases as this mode evolves into the ZB (or out-of-phase) plasmon of a 1D wire superlattice. The lowest ZC mode (indicated by solid circles) tracks along the CM mode in Fig. 7 and, as discussed above, goes through a minimum at the transition from 2D to $1 \mathrm{D}$ behaviour. This behaviour is qualitatively similar to that observed21.22, although it should be noted that in 
the experiments the average density of the gas decreased as a function of the gating potential, whereas our results are for a constant density. The CM mode crosses the upper branch of the second $\mathrm{ZB}$ mode at $V_{0} / E_{f} \simeq 20$ and the pair then defines the CM band with its dipole induced dispersion. For the higher modes we see the merging of successive pairs of ZC and ZB modes in the 1D limit. The increasing frequency of these modes is once again due to the increasing curvature of the confining potential. Fig. 14 extends the variation of the mode frequencies with $V_{0}$ into the localized $1 \mathrm{D}$ regime which is beyond that given previously $7 \mathrm{id}$. In particular, we do not find the unphysical result claimed in Ref. [10] that the system does not support plasmons in the strong localization limit.

\section{CONCLUSIONS}

In this paper, we have demonstrated that the Thomas-Fermi-Dirac-von Weizsäcker approximation provides a realistic description of the collective excitations in a modulated 2DEG. We have presented a detailed investigation of the equilibrium properties, and have numerically mapped out the parameter space that defines the transition from $2 \mathrm{D}$ to $1 \mathrm{D}$ behavior. We have also calculated the plasmon dispersions for propagation along and across the modulation direction in the weak, moderate and strong modulation limits. In agreement with earlier work on this problem, the modulation of the equilibrium density leads to the appearance of gaps in the plasmon dispersion in the direction of the modulation; as the modulation becomes stronger the bands become narrower and the gaps larger. For propagation in a direction perpendicular to the direction of modulation, we have shown that the long-wavelength 2D plasmon is unaffected by the modulation potential. However, at shorter wavelengths, the plasmon dispersions exhibit interesting behaviour as a function of $q_{y}$ and the explicit calculation of the mode densities provides a more complete understanding of the physical nature of these excitations.

The power absorption in the long-wavelength limit has also been calculated for a range of modulation potentials. We have found that the oscillator strength is predominantly in the Drude peak for weak modulation and shifts to higher dipole modes as the modulation is increased. The reduction of the Drude peak is one useful indicator to gauge the effective dimensionality of the system. We have also shown that the $2 \mathrm{D}$ bulk plasmon at $q=2 \pi / a$ evolves continuously with increasing modulation into the CM mode of a quantum wire superlattice. The frequency of the mode at first decreases, and then passes through a minimum as the 2DEG makes the transition from a continuous charge distribution to an array of isolated wires. This minimum is a second signature of the $2 \mathrm{D} \rightarrow 1 \mathrm{D}$ crossover. At large modulations, the confining potential is effectively harmonic, and the FIR oscillator strength resides in the CM mode in accord with the generalized Kohn theorem.

We are at present completing a detailed analysis of the magnetoplasma excitations in laterally modulated 2D systems. These results will be reported elsewhere.

\section{ACKNOWLEDGMENTS}

The work was supported by a grant from the Natural Sciences and Engineering Research Council of Canada. We would like to thank Dr. D. A. W. Hutchinson for useful discussions. 


\section{APPENDIX A: CALCULATION OF PLASMON BAND GAPS}

In this appendix, we determine the eigenvalue spectrum of a laterally modulated 2DEG in the weak modulation regime. The eigenvalue problem that we wish to solve is

$$
\begin{aligned}
\omega^{2} c_{G} & =\sum_{G^{\prime} G^{\prime \prime}} B_{G G^{\prime}} \tilde{M}_{G^{\prime} G^{\prime \prime}} c_{G^{\prime \prime}} \\
& =\sum_{G^{\prime}} N_{G G^{\prime}} c_{G^{\prime}}
\end{aligned}
$$

with

$$
N_{G G^{\prime}} \equiv \sum_{G^{\prime \prime}} B_{G G^{\prime \prime}} \tilde{M}_{G^{\prime \prime} G^{\prime}}
$$

In the absence of an external modulation, the $N$-matrix is diagonal. Denoting the matrices in this limit by a superscript 0, we have

$$
\begin{aligned}
B_{G G^{\prime}}^{0} & =\frac{1}{2}\left(\left(q_{x}+G\right)^{2}+q_{y}^{2}\right) \delta_{G G^{\prime}} \\
\tilde{M}_{G G^{\prime}}^{0} & =\left[\frac{4 \pi \bar{n}_{2 D}}{\sqrt{\left(q_{x}+G\right)^{2}+q_{y}^{2}}}+4 C_{1} \bar{n}_{2 D}-\frac{3}{2} C_{3} \sqrt{\bar{n}_{2 D}}+\frac{\lambda_{w}}{2}\left(\left(q_{x}+G\right)^{2}+q_{y}^{2}\right)\right] \delta_{G G^{\prime}} .
\end{aligned}
$$

With these results, we have at $q_{y}=0$,

$$
\begin{aligned}
N_{G G^{\prime}}^{0} & =\sum_{G^{\prime \prime}} B_{G G^{\prime \prime}}^{0} \tilde{M}_{G^{\prime \prime} G^{\prime}}^{0} \\
& =\left[2 \pi \bar{n}_{2 D}\left|q_{x}+G\right|+\left(2 C_{1} \bar{n}_{2 D}-\frac{3}{4} C_{3} \sqrt{\bar{n}_{2 D}}\right)\left(q_{x}+G\right)^{2}+\frac{\lambda_{w}}{4}\left(q_{x}+G\right)^{4}\right] \delta_{G G^{\prime}} \\
& \equiv \omega_{0}^{2}\left(q_{x}+G\right) \delta_{G G^{\prime}} .
\end{aligned}
$$

This equation gives the plasmon frequency in Sec. IVA. With $G$ taking on all possible values, $q_{x}$ can be restricted to the first Brillouin zone, $-\pi / a \leq q_{x} \leq \pi / a$.

The introduction of an external modulation lifts the degeneracies in the uniform gas spectrum at the zone center $\left(q_{x}=0\right)$ and at the zone boundary $\left(q_{x}=\pi / a\right)$. At the zone center (ZC), the reciprocal lattice vectors coupling degenerate modes are given by

$$
q_{x}=0, \quad G_{1}=n \frac{\pi}{a}, G_{2}=-n \frac{\pi}{a},
$$

where $n$ is an even integer, while at the zone boundary (ZB)

$$
q_{x}=\frac{\pi}{a}, \quad G_{1}=(n-1) \frac{\pi}{a}, \quad G_{2}=-(n+1) \frac{\pi}{a},
$$

where $n$ is an odd integer. At either ZC or ZB, $\left|q_{x}+G_{1}\right|=\left|q_{x}+G_{2}\right|=n \pi / a \equiv q_{n}$ and $\left(G_{1}-G_{2}\right)=2 n \pi / a=2 q_{n}$.

We now apply degenerate perturbation theory to obtain first-order corrections to the frequencies at these points in the first Brillouin zone. Retaining only the two degenerate modes of interest, we have the pair of equations 


$$
\begin{aligned}
& \omega^{2} c_{G_{1}}=N_{G_{1} G_{1}}^{0} c_{G_{1}}+N_{G_{1} G_{2}}^{1} c_{G_{2}} \\
& \omega^{2} c_{G_{2}}=N_{G_{2} G_{1}}^{1} c_{G_{1}}+N_{G_{2} G_{2}}^{0} c_{G_{2}},
\end{aligned}
$$

where $N_{G_{1} G_{2}}^{1}=N_{G_{2} G_{1}}^{1}$ is the lowest order off-diagonal correction to the $N$-matrix. It is given by

$$
N_{G_{1} G_{2}}^{1}=B_{G_{1} G_{1}}^{0} \tilde{M}_{G_{1} G_{2}}^{1}+B_{G_{1} G_{2}}^{1} \tilde{M}_{G_{2} G_{2}}^{0}
$$

where

$$
\begin{aligned}
\tilde{M}_{G_{1} G_{2}}^{1} & =\frac{8 \pi}{q_{n}} \sqrt{\frac{\bar{n}_{2 D}}{a}} \bar{\psi}_{0}\left[2 q_{n}\right]+\frac{4 C_{1}}{\sqrt{a}} \bar{n}_{0}\left[2 q_{n}\right]-\frac{3 C_{3}}{\sqrt{a}} \bar{\psi}_{0}\left[2 q_{n}\right]-\frac{2 \lambda_{w}}{\sqrt{\bar{n}_{2 D} a}} q_{n}^{2} \bar{\psi}_{0}\left[2 q_{n}\right] \\
B_{G_{1} G_{2}}^{1} & =-\frac{2}{\sqrt{\bar{n}_{2 D}}} q_{n}^{2} \bar{\psi}_{0}\left[2 q_{n}\right] .
\end{aligned}
$$

In the above, $\bar{\psi}_{0}\left[2 q_{n}\right]$ and $\bar{n}_{0}\left[2 q_{n}\right]$ denote the $n$-th Fourier components of the groundstate wavefunction and charge density respectively. Replacing $\bar{\psi}_{0}\left[2 q_{n}\right]$ by $\bar{n}_{0}\left[2 q_{n}\right] / 2 \sqrt{\bar{n}_{2 D}}$, Eq. (A8) can be cast in the form

$$
N_{G_{1} G_{2}}^{1}=\left[-\omega_{0}^{2}\left(q_{n}\right)+\frac{3}{8} C_{3} \sqrt{\bar{n}_{2 D}} q_{n}^{2}-\frac{3}{4} \lambda_{w} q_{n}^{4}\right]\left[\frac{\bar{n}_{0}\left[2 q_{n}\right]}{\bar{n}_{0}\left[2 q_{0}\right]}\right] .
$$

This implies that the eigenvalues of (A7) are given by

$$
\begin{aligned}
\omega_{ \pm}^{2}\left(q_{n}\right) & =N^{0}\left[q_{n}\right] \pm\left|N^{1}\left[q_{n}\right]\right| \\
& =\omega_{0}^{2}\left(q_{n}\right) \pm\left|\omega_{0}^{2}\left(q_{n}\right)-\frac{3}{8} C_{3} \sqrt{\bar{n}_{2 D}} q_{n}^{2}+\frac{3}{4} \lambda_{w} q_{n}^{4}\right|\left|\frac{\bar{n}_{0}\left[2 q_{n}\right]}{\bar{n}_{0}\left[2 q_{0}\right]}\right|,
\end{aligned}
$$

where $N^{0}\left[q_{n}\right] \equiv N_{G_{1} G_{1}}^{0}$ and $N^{1}\left[q_{n}\right] \equiv N_{G_{1} G_{2}}^{1}$. The size of the gap at the ZC or ZB is given by $2\left|N_{1}\left[q_{n}\right]\right|$. We have tested the validity of (A11), and in the regime where the perturbative results are valid, we find excellent agreement between the analytical and numerical calculations as illustrated in Fig. 14. In the Hartree approximation, we obtain the simple relation

$$
\omega_{ \pm}^{2}\left(q_{n}\right)=\omega_{0}^{2}\left(q_{n}\right)\left(1 \pm\left|\frac{\bar{n}_{0}\left[2 q_{n}\right]}{\bar{n}_{0}\left[2 q_{0}\right]}\right|\right) .
$$

This result is entirely consistent with the one obtained by Krasheninnikov and Chaplik in the long-wavelength limit.

\section{APPENDIX B: DETERMINATION OF LONG WAVELENGTH DISPERSIONS}

A persistent feature of the dispersion relations we have calculated (see Figs. (1, 8 and 11), is that the long-wavelength limit of the lowest plasmon has the characteristic $\sqrt{q}$ dependence

of the 2D-bulk plasmon, regardless of the modulation. In this section, we show analytically that the long-wavelength behaviour of the lowest plasmon branch is $\sqrt{q}$ for any modulation amplitude. 
Once again, the eigenvalue problem that we are solving has the form

$$
\begin{aligned}
\omega^{2} c_{G} & =\sum_{G^{\prime} G^{\prime \prime}} B_{G G^{\prime}} \tilde{M}_{G^{\prime} G^{\prime \prime}} c_{G^{\prime \prime}} \\
& =\frac{1}{\lambda_{w}} \sum_{G^{\prime} G^{\prime \prime}} h_{G G^{\prime}} \tilde{M}_{G^{\prime} G^{\prime \prime}} c_{G^{\prime \prime}},
\end{aligned}
$$

where $h_{G G^{\prime}}$ is the Hamiltonian which, for $q_{y}=0$, determines the groundstate von Weizsäcker wave function $\bar{\psi}_{0}[G]$. In the long-wavelength limit, the dominant contribution to the $\tilde{M}$ matrix comes from the $G^{\prime \prime}=0$ component of the Hartree matrix in Eq. (39),

$$
\lim _{q \rightarrow 0} \tilde{M}_{G G^{\prime}} \simeq \lim _{q \rightarrow 0} M_{G G^{\prime}}^{H} \simeq \frac{4 \pi}{q a} \bar{\psi}_{0}[G] \bar{\psi}_{0}\left[G^{\prime}\right]
$$

With this result, and noting that $\sum_{G^{\prime}} h_{G G^{\prime}} \bar{\psi}_{0}\left[G^{\prime}\right]=\varepsilon_{0}(\mathbf{q}) \bar{\psi}_{0}[G]$, Eq. (B1) can be written as

$$
\lambda_{w} \omega^{2} c_{G}=\frac{4 \pi}{q a} \varepsilon_{0}(\mathbf{q}) \bar{\psi}_{0}[G] \sum_{G^{\prime \prime}} \bar{\psi}_{0}\left[G^{\prime \prime}\right] c_{G^{\prime \prime}} .
$$

The eigenvalues $\omega^{2}$ of (B3) are thus given by

$$
\omega^{2}\left(q_{x}, q_{y}\right)=\frac{4 \pi \bar{n}_{2 D}}{q} \varepsilon_{0}(\mathbf{q})
$$

since $\bar{n}_{2 D}=a^{-1} \sum_{G}\left|\bar{\psi}_{0}[G]\right|^{2}$. For small $\mathbf{q}$ we have $\varepsilon_{0}(\mathbf{q}) \simeq \frac{\lambda_{w}}{2}\left(\frac{q_{x}^{2}}{m_{x}}+q_{y}^{2}\right)$, where $m_{x}$ is the effective band mass due to the confining potential in the modulation direction. Of interest here is the determination of the dispersions in both the $q_{x}=0, q_{y} \rightarrow 0$ and $q_{y}=0, q_{x} \rightarrow 0$ limits. In these two cases we have

$$
\begin{gathered}
\omega^{2}\left(q_{x}=0, q_{y}\right) \simeq 2 \pi \bar{n}_{2 D} q_{y} \\
\omega^{2}\left(q_{x}, q_{y}=0\right) \simeq \frac{2 \pi \bar{n}_{2 D}}{m_{x}} q_{x} .
\end{gathered}
$$

Eq. (B5) illustrates the fact that for any modulation, the long-wavelength behaviour of the $2 \mathrm{D}$ plasmon is $\sqrt{q_{y}}$. Eq. (B6) on the other hand indicates that the $\sqrt{q_{x}}$ dispersion is suppressed with increasing modulation as a result of the effective mass $m_{x}$. 


\section{REFERENCES}

${ }^{1}$ Interfaces, Quantum Wells, and Superlattices, edited by C. R. Leavens and Roger Taylor (Plenum, New York, 1988).

2 Physics and Technology of Submicron Structures, vol 38 of Springer Series in Solid-State Science, edited by H. Heinrich, G. Bauer and F. Kuchar (Springer-Verlag, Berlin, 1988).

${ }^{3}$ Quantum Coherence in Mesoscopic Systems, edited by Bernhard Kramer (Plenum, New York, 1991).

${ }^{4}$ Nanostructures and Mesoscopic Systems, edited by W.P. Kirk and M.A. Reed (Academic Press, Boston, 1992).

${ }^{5}$ M. V. Krasheninnikov and A. V. Chaplik, Fiz. Tekh. Poluprovodn. 15, 32 (1981) [Sov. Phys.-Semicond. 15, 19 (1981)].

${ }^{6}$ S. Das Sarma and W. Y. Lai, Phys. Rev. B 32, 1401 (1985).

${ }^{7}$ G. Eliasson, P. Hawrylak, J.-W. Wu, and J.J. Quinn, Solid State Commun. 60, 3 (1986).

${ }^{8}$ G. Eliasson, J.-W. Wu, P. Hawrylak, and J.J. Quinn, Solid State Commun. 60, 41 (1986).

${ }^{9}$ W. Y. Lai, A. Kobayashi, and S. Das Sarma, Phys. Rev. B 34, 7380 (1986).

${ }^{10}$ V. Cataudella and V. M. Ramaglia, Phys. Rev. B 38, 1828 (1988).

${ }^{11}$ Y. Zhu and S. Z. Zhou, J. Phys. C 21, 3063 (1988).

${ }^{12}$ W. Que and G. Kirczenow, Phys. Rev. B 39, 5998 (1989).

${ }^{13}$ U. Wulf, E. Zeeb, P. Gies, R.R. Gerhardts and W. Hanke, Phys. Rev. B 41, 3113 (1990).

${ }^{14}$ U. Wulf, E. Zeeb, P. Gies, R.R. Gerhardts and W. Hanke, Phys. Rev. B 42, 7637 (1990).

${ }^{15}$ C. Dahl, Phys. Rev. B 41, 5763 (1990).

${ }^{16}$ V. B. Shikin, T. Demel' and D Heitmann, Sov. Phys. JETP 69, 797 (1990).

${ }^{17}$ P. W. Park, A. H. MacDonald and W. L. Schaich, Phys. Rev. B 46, 12635 (1992).

${ }^{18}$ W. L. Schaich, P.W. Park and A. H. MacDonald, Phys. Rev. B 46, 12643 (1992).

${ }^{19}$ V. Gudmundsson, A. Brataas, P. Grambow, B. Meurer, T. Kurth and D. Heitmann, Phys. Rev. B 51, 17744 (1995).

${ }^{20}$ U. Mackens, D. Heitmann, L. Prager, and J. P. Kotthaus, Phys. Rev. Lett. 53, 1485 (1984).

${ }^{21}$ W. Hansen, M. Horst, J.P. Kotthaus, U. Merkt, Ch. Sikorski and K. Ploog, Phys. Rev. Lett. 58, 2586 (1987).

${ }^{22}$ F. Brinkop, W. Hansen, J.P. Kotthaus and K. Ploog, Phys. Rev. B 37, 6547 (1988).

${ }^{23}$ T. Demel, D. Heitmann, P. Grambow and K. Ploog, Phys. Rev. B 38, 12732 (1988).

${ }^{24}$ H. Drexler, W. Hansen, J.P. Kotthaus, M. Holland and S.P. Beaumont, Phys. Rev. B 46, 12849 (1992).

${ }^{25}$ G. Hertel, H. Drexler, W. Hansen, A. Schmeller, J. P. Kotthaus, M. Holland, and S. P. Beaumont, Solid-State Electronics, 37, 1289 (1994).

${ }^{26}$ C. Steinbach, R. Krane, G. Biese, C. Schüller, D. Heitmann, and K. Eberl, Phys. Rev. B 54, R14281 (1996).

${ }^{27}$ D. Heitmann, K. Bollweg, V. Gudmundsson, T. Kurth and S.P. Reige, Physica E 1, 204 (1997).

${ }^{28}$ W. R. Frank, A. O. Govorov, J.P. Kotthaus, C. Steinbach, V. Gudmundsson, W. Hansen and M. Holland, Phys. Rev. B 55, R1950 (1997).

${ }^{29}$ W. L. Schaich and J. F. Dobson, Phys. Rev. B 49, 14700 (1994).

${ }^{30}$ F. Forstmann and R. R. Gerhardts, Metal Optics Near the Plasma Frequency (SpringerVerlag, Berlin, 1986). 
${ }^{31}$ A. L. Fetter, Ann. Phys. (N.Y.) 88, 1 (1974); A. Eguiluz and J. J. Quinn, Phys. Rev. B 14, 1347 (1976); S. Das Sarma and J. J. Quinn, ibid. 20, 4872 (1979).

${ }^{32}$ E. Zaremba and H. C. Tso, Phys. Rev. B 49, 8147 (1994).

${ }^{33}$ E. Zaremba and H. C. Tso, in Electronic Density Functional Theory: Recent Progress and New Directions, ed. by J.F. Dobson, G. Vignale and M.P. Das (Plenum, New York, 1998).

${ }^{34}$ E. Zaremba, Phys. Rev. B 53, R10512 (1996).

${ }^{35}$ A. V. Chaplik, Zh. Eksp. Teor. Fiz. 60, 1845 (1971) [Sov. Phys. JETP 33, 947 (1971)]; F. Stern, Phys. Rev. Lett. 30, 278 (1973).

${ }^{36}$ A. Chizmeshya and E. Zaremba, Phys. Rev. B 37, 2805 (1988).

${ }^{37}$ The mode is actually gapless at $q_{y}=0$ for all $q_{x}$ values (see the "1-2" branch in Fig. (11). Fig. 12 shows the $q_{y}$-dispersion for the limiting values of $q_{x}=0$ and $q_{x}=\pi / a$.

${ }^{38}$ For a single wire of width $a$, the plasmon frequency behaves as ${ }^{6}$. a pair of such wires, the out-of-phase plasmon has a linear dispersion 11 . The $q_{x}=\pi / a$ mode should behave in a similar way.

${ }^{39}$ L. Brey, N.F. Johnson and B.I. Halperin, Phys. Rev. B 40, 10647 (1989).

${ }^{40}$ S.K. Yip, Phys. Rev. B 43, 1707 (1991).

${ }^{41}$ J.F. Dobson, Phys. Rev. Lett. 73, 2244 (1994). 


\section{FIGURES}

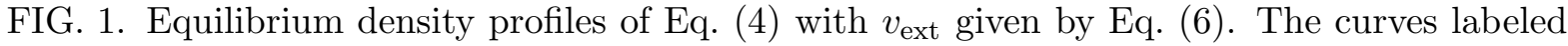
by (a), (b), (c), and (d) correspond to $V_{0} / E_{f}=6.4,19.1,28.6$ and 41.4, respectively. The mean density in all cases is $\bar{n}_{2 D}=0.1$.

FIG. 2. "Phase"-diagram for the $2 \mathrm{D} \rightarrow 1 \mathrm{D}$ transition. The solid and dashed curves include all interactions with $\bar{n}_{2 D}=0.1$ and $\bar{n}_{2 D}=1.0$ respectively. The open circles correspond to the noninteracting phase curve (i.e. a confined noninteracting Fermi gas) which is independent of the average $2 \mathrm{D}$ density.

FIG. 3. The calculated TFDW energy bands along the direction of modulation. This figure is evaluated for a moderate modulation corresponding to "(b)" in Fig. 1.

FIG. 4. The dispersion of mode frequencies with wave vector $q_{x}$ for the weakly modulated 2DEG. The solid curve is for the uniform 2DEG. A full explanation of the encircled numbers is given in the text.

FIG. 5. The dispersion of mode frequencies with wave vector $q_{y}$ for the weakly modulated 2DEG. Here $q_{x}=0$ (solid circles), and $q_{x}=\pi / a$ (open circles). A full explanation of the encircled numbers is given in the text.

FIG. 6. The mode densities in the weak modulation regime for various bands. The left panels are evaluated at fixed $q_{y}=0$ and the right panels at evaluated at $q_{y}=3 \pi / a$. The sequence for the panels from top to bottom is $q_{x}=0, q_{x}=\pi / a$, and $q_{x}=0$. See the text for full details.

FIG. 7. Power absorption as a function of frequency. The curves are ordered from weakest (bottom) to strongest (top) modulation and are offset by $\Delta V_{0}=1.0$ a.u. The labels $(a),(b),(c)$ and $(d)$ are consistent with the notation adopted in Figs. 1 and 2.

FIG. 8. As in Fig. 4, but for moderate modulation.

FIG. 9. As in Fig. 5, but for moderate modulation.

FIG. 10. As in Fig. 6, but for moderate modulation.

FIG. 11. As in Fig. 1 , but for strong modulation.

FIG. 12. As in Fig. , but for strong modulation. 
FIG. 13. As in Fig. 6, but for strong modulation.

FIG. 14. Zone boundary (open circles) and zone center (filled circles) frequencies (in a.u.) at $q_{y}=0$ as a function of the strength of the modulating potential. The solid curves are the solutions given by Eq. (A11) for $n=1$.

FIG. 15. The first three Fourier coefficients of the equilibrium charge density as a function of modulation strength. The curves are normalized with respect to the average areal density $\bar{n}_{2 D}$. Here $G_{n}=2 n \pi / a$, with $n=1$ corresponding to the figure inset (solid line), $n=2$ the short-dash-long-dash curve and $n=3$ the dashed curve. 


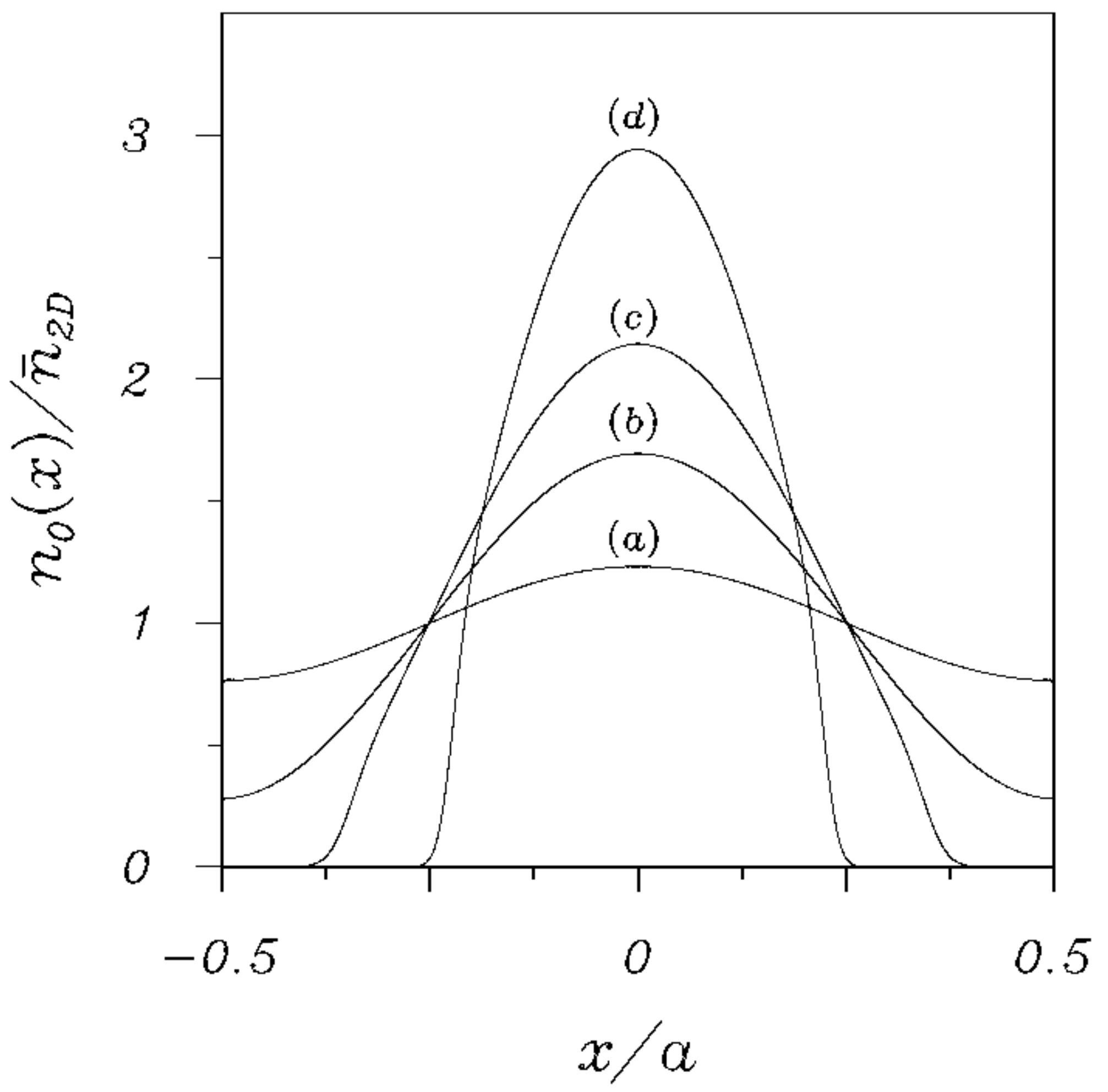


Fig. 2

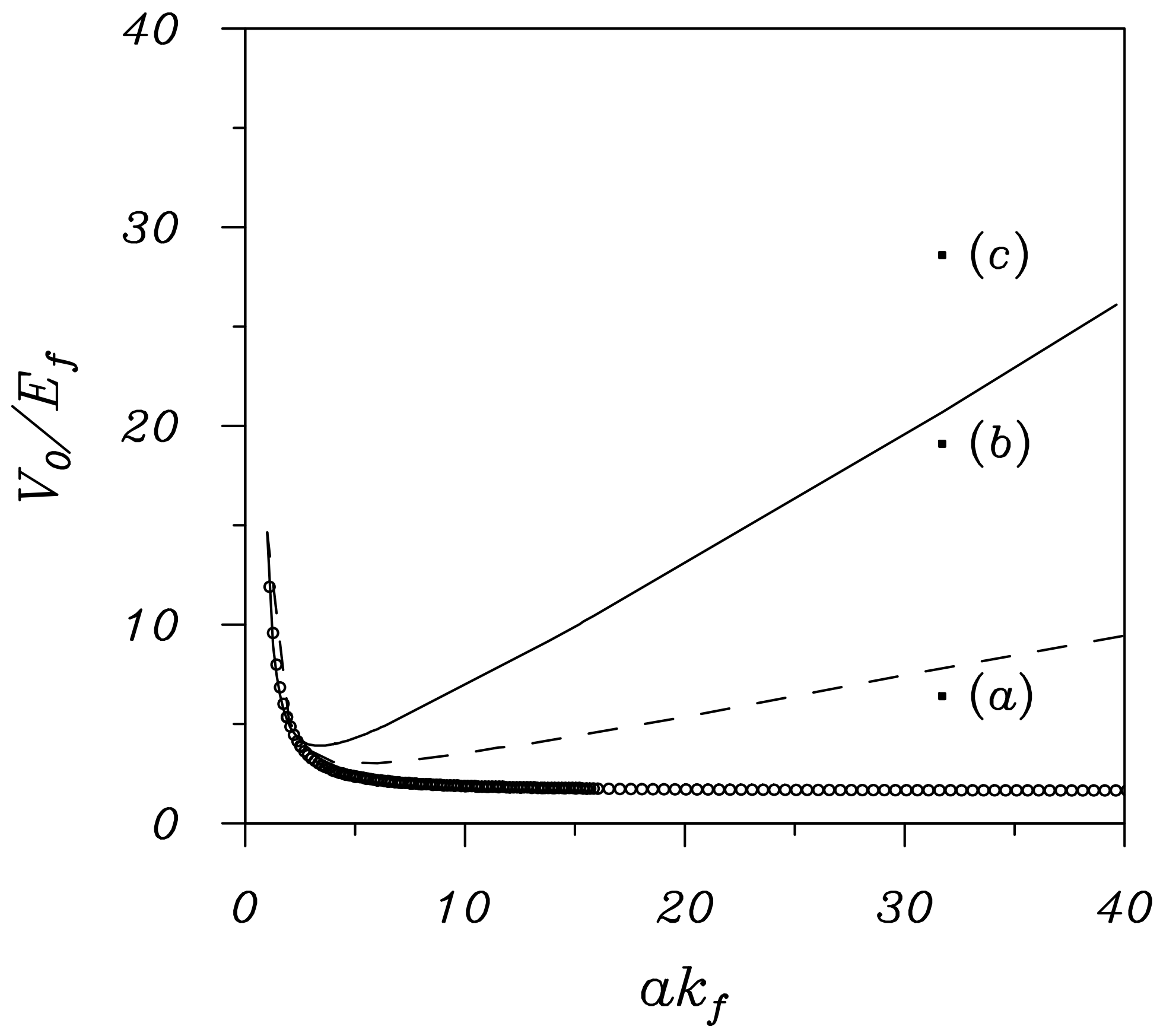




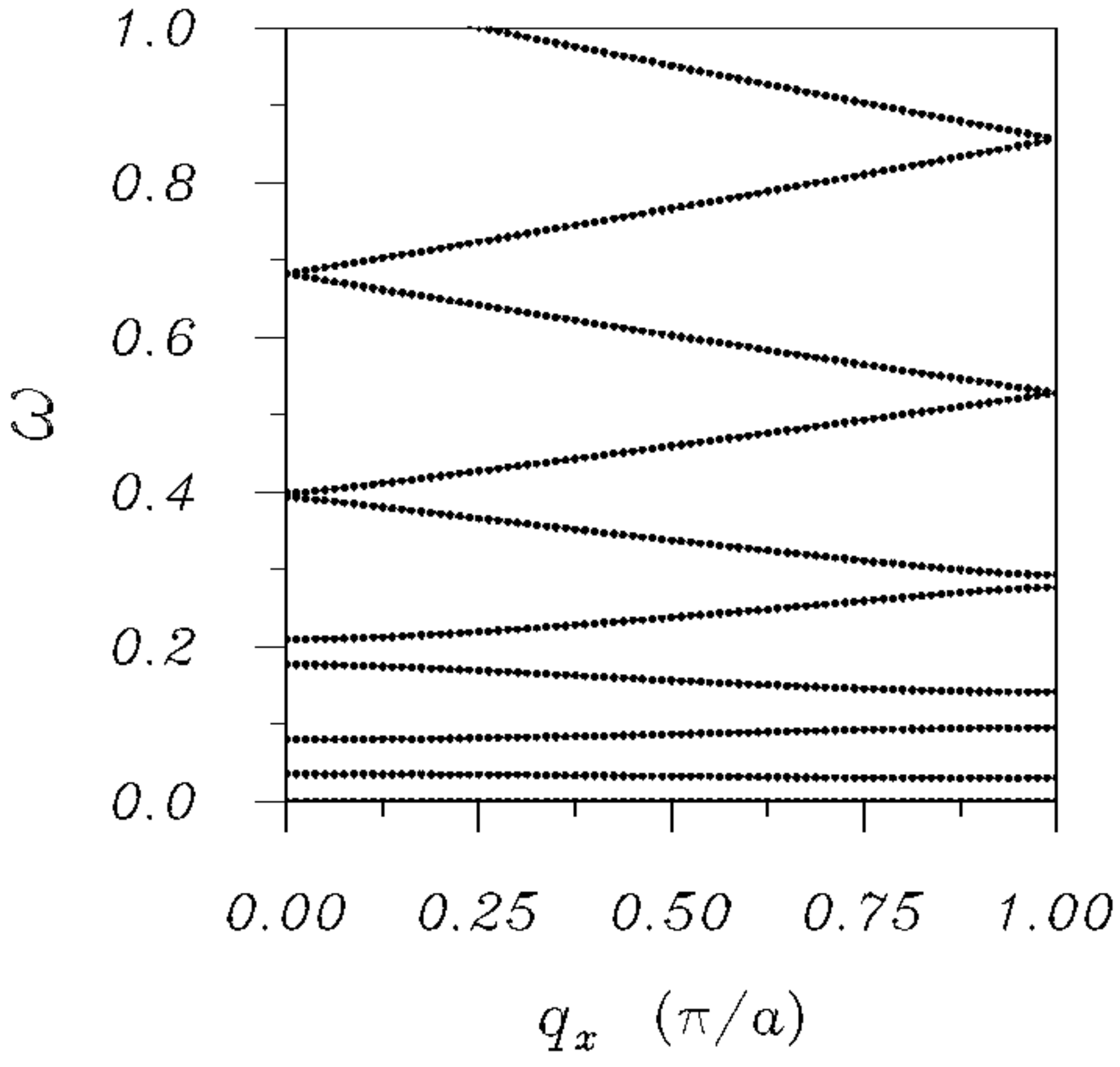




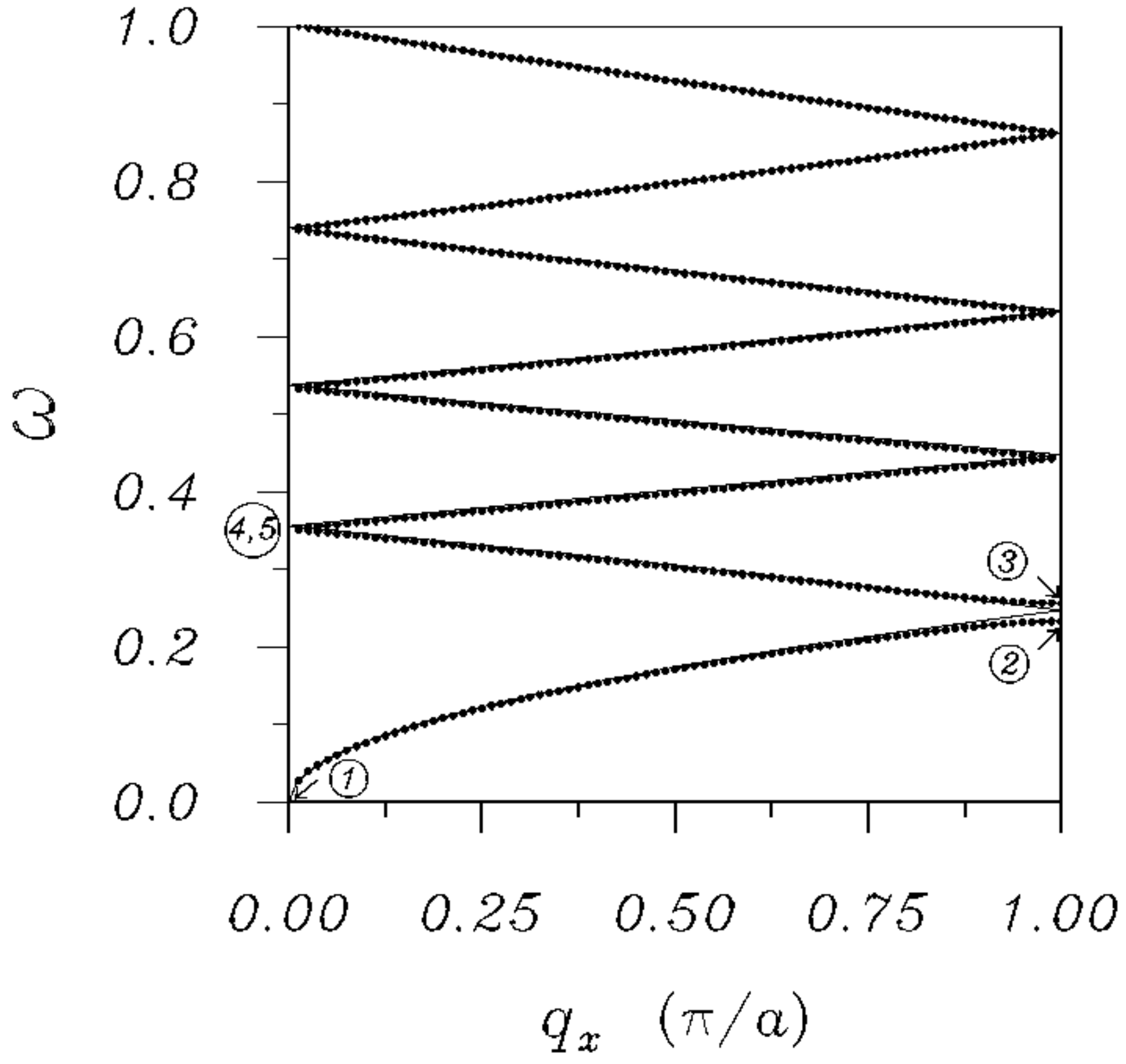




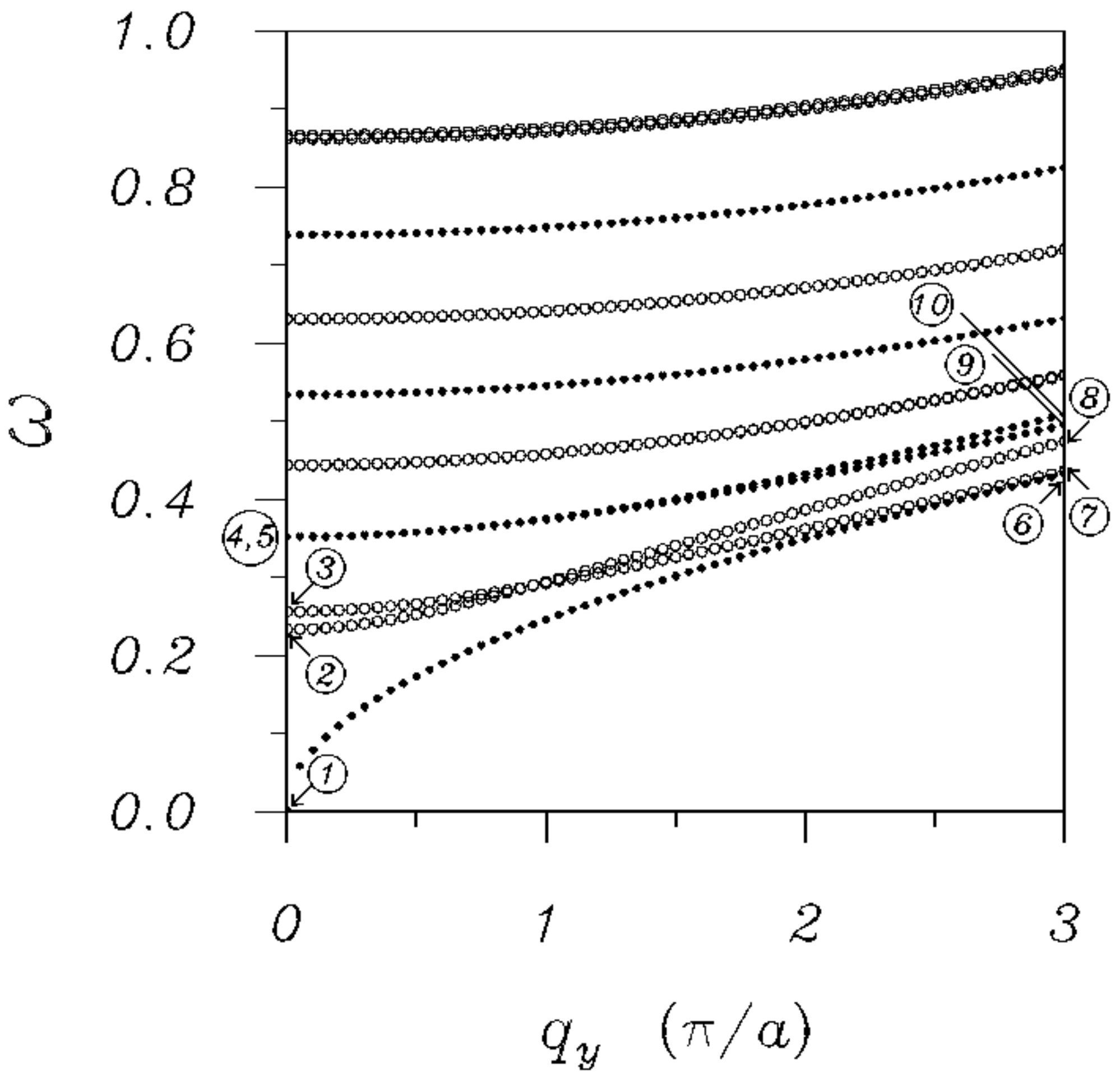


Fig. 6
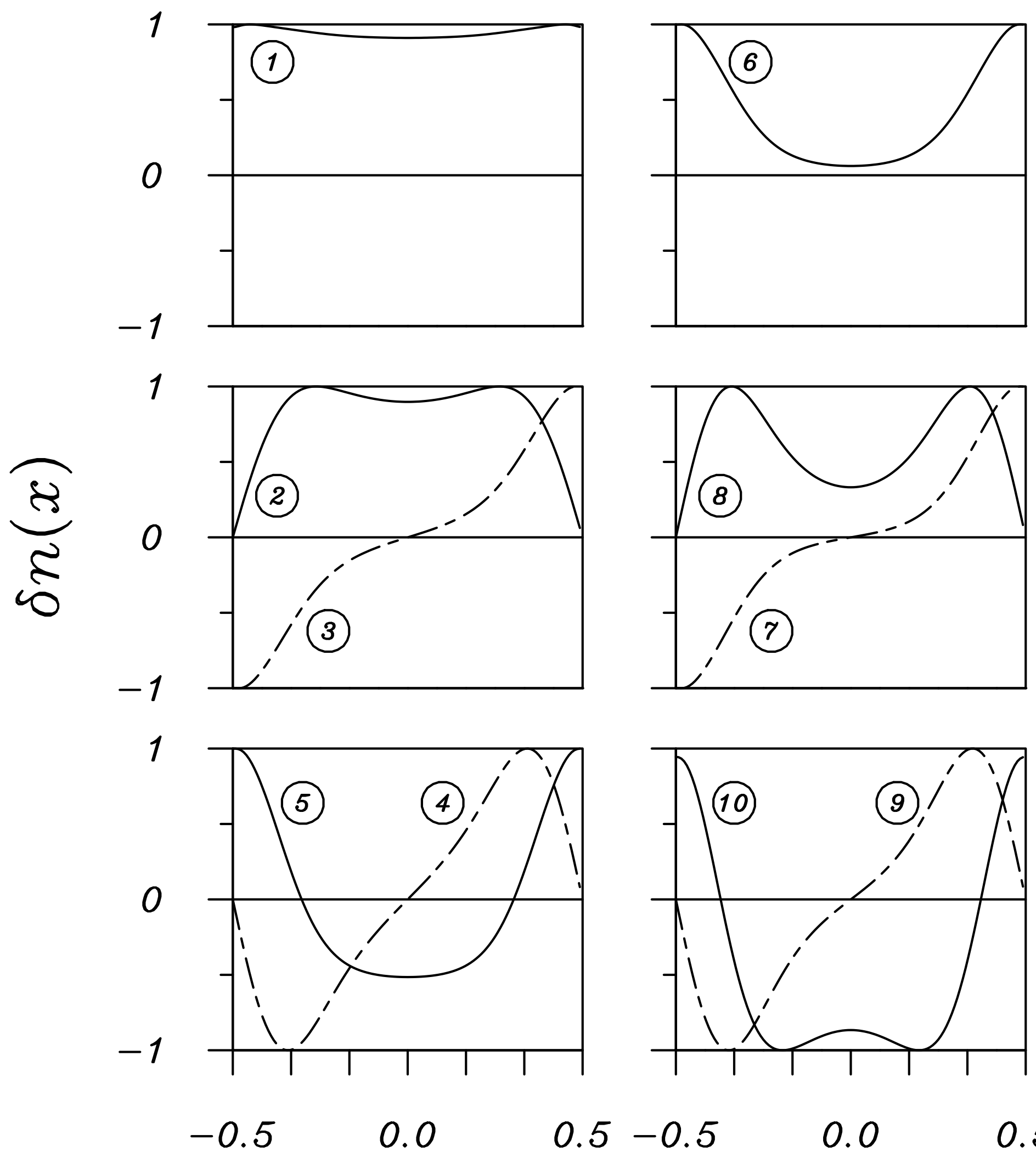

0.0

0.5 
Fig. 7

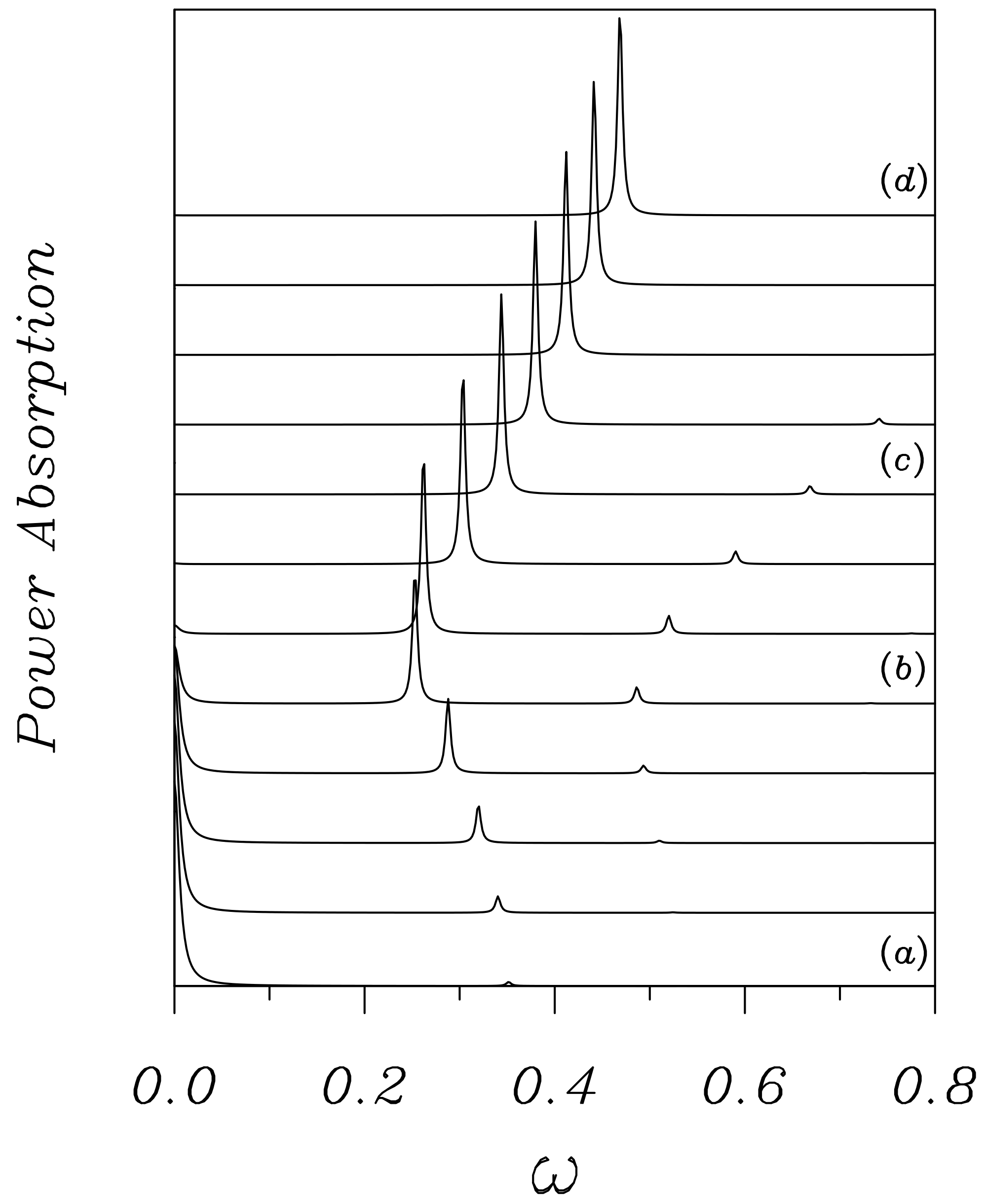




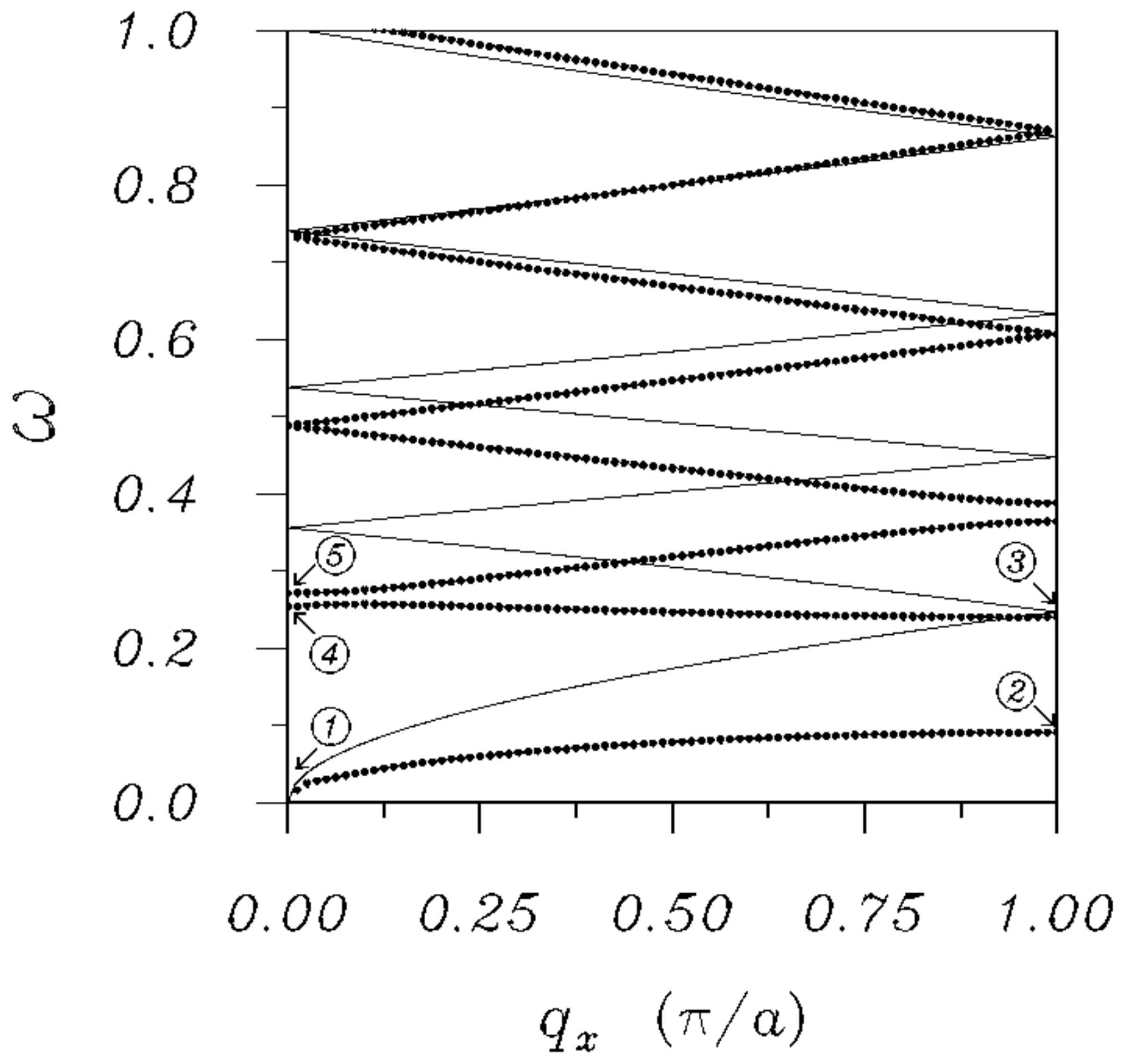




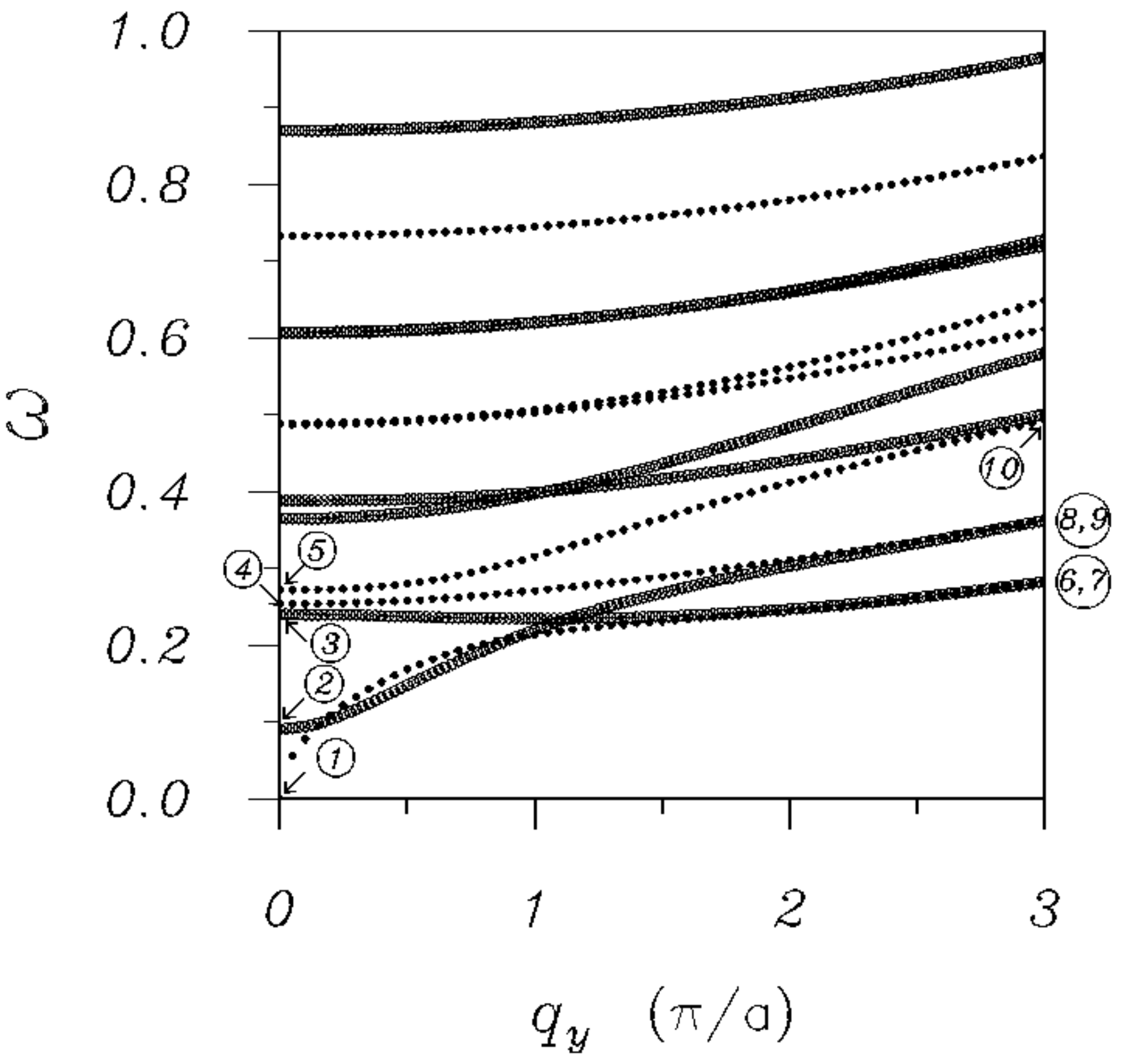


Fig. 10
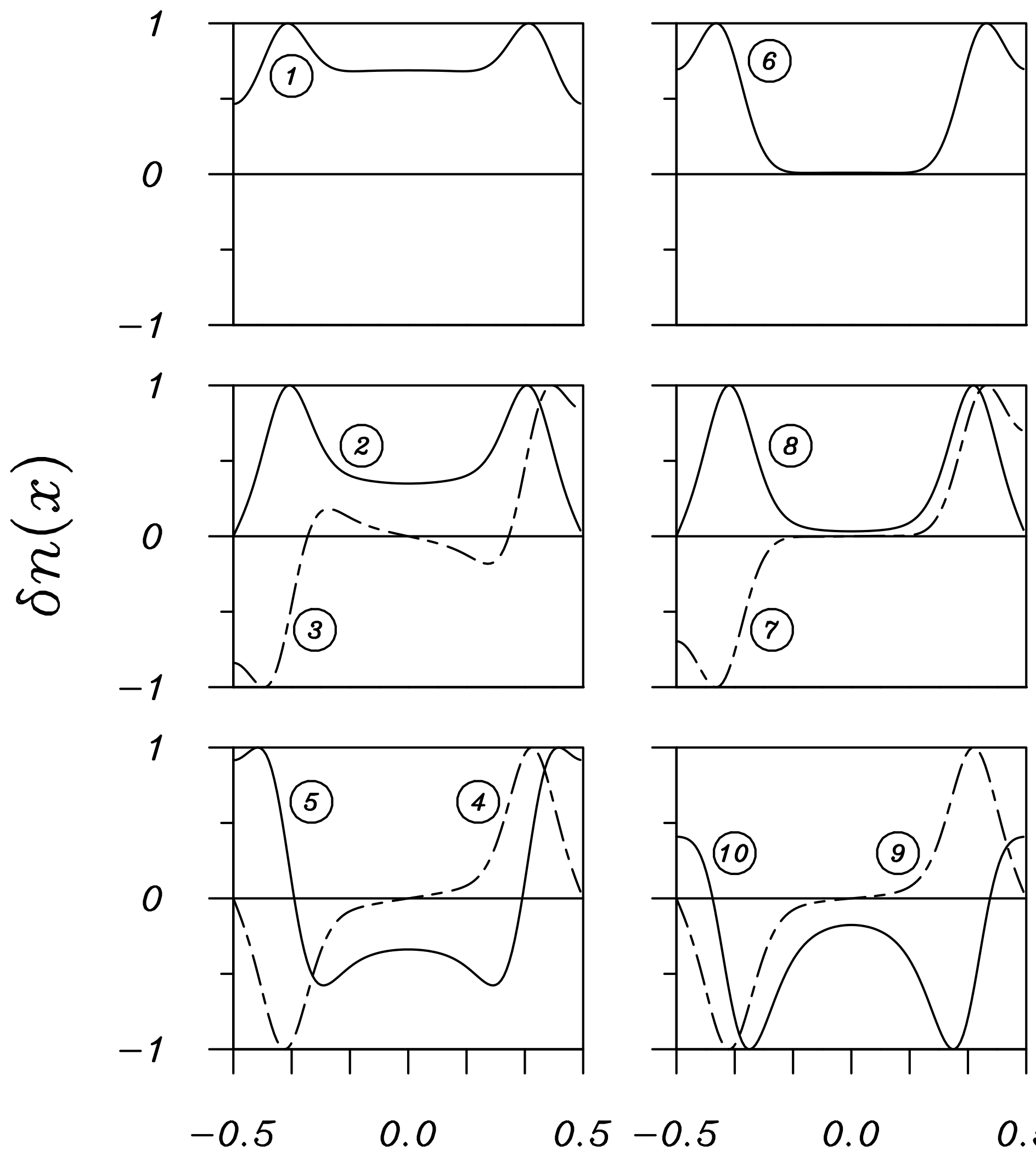

0.0

0.5 


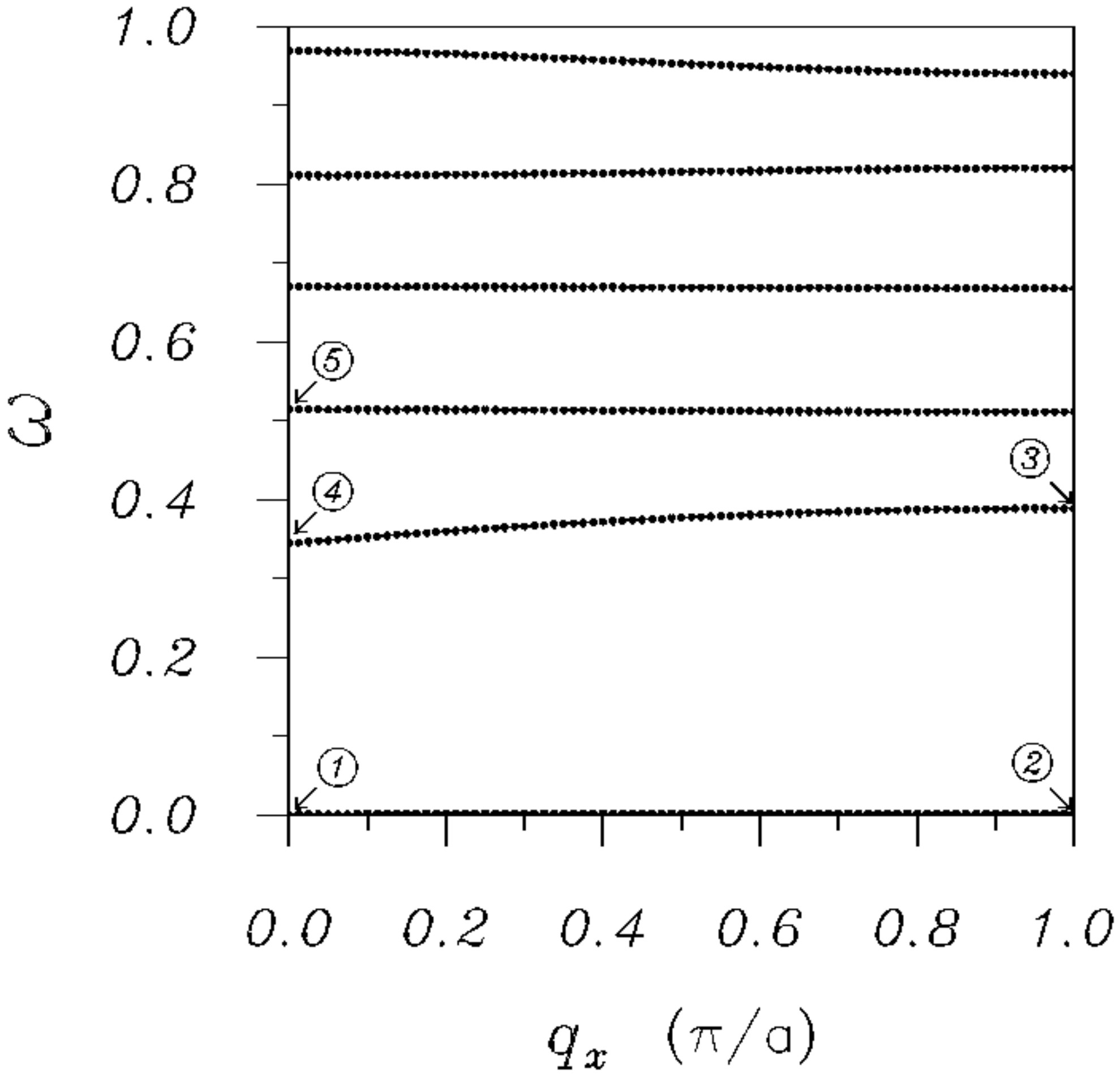




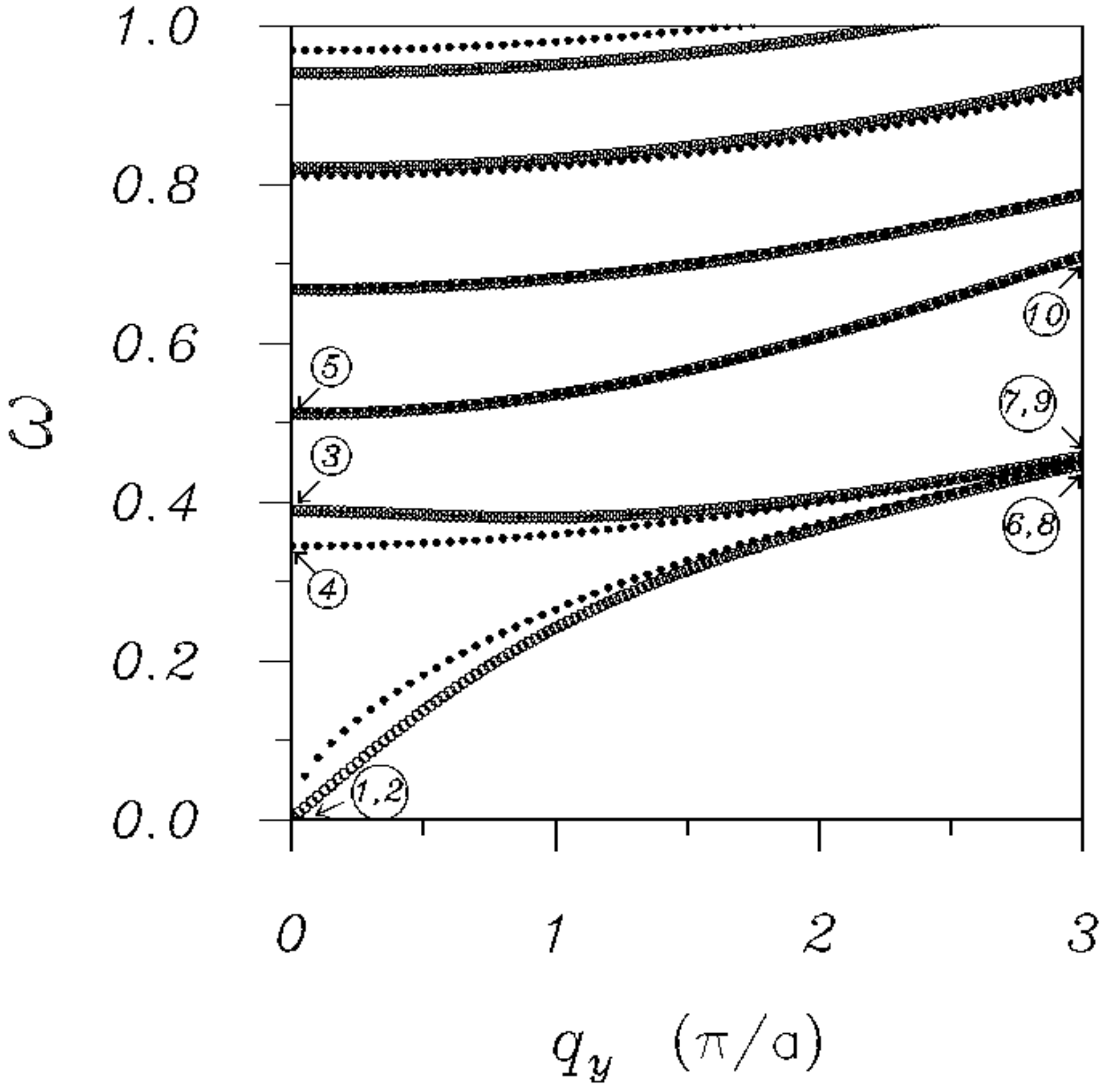


Fig. 13
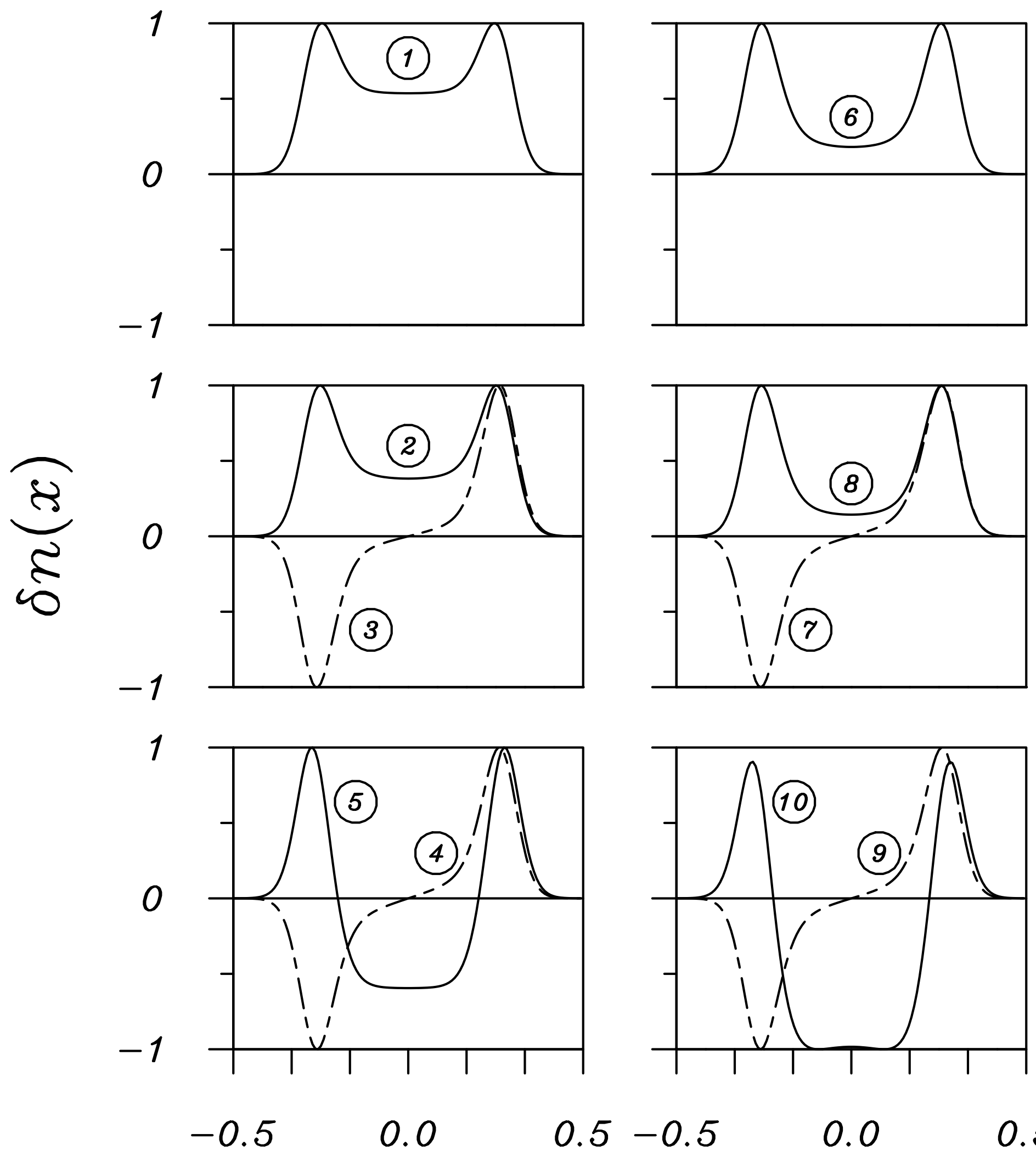

0.0

0.5 


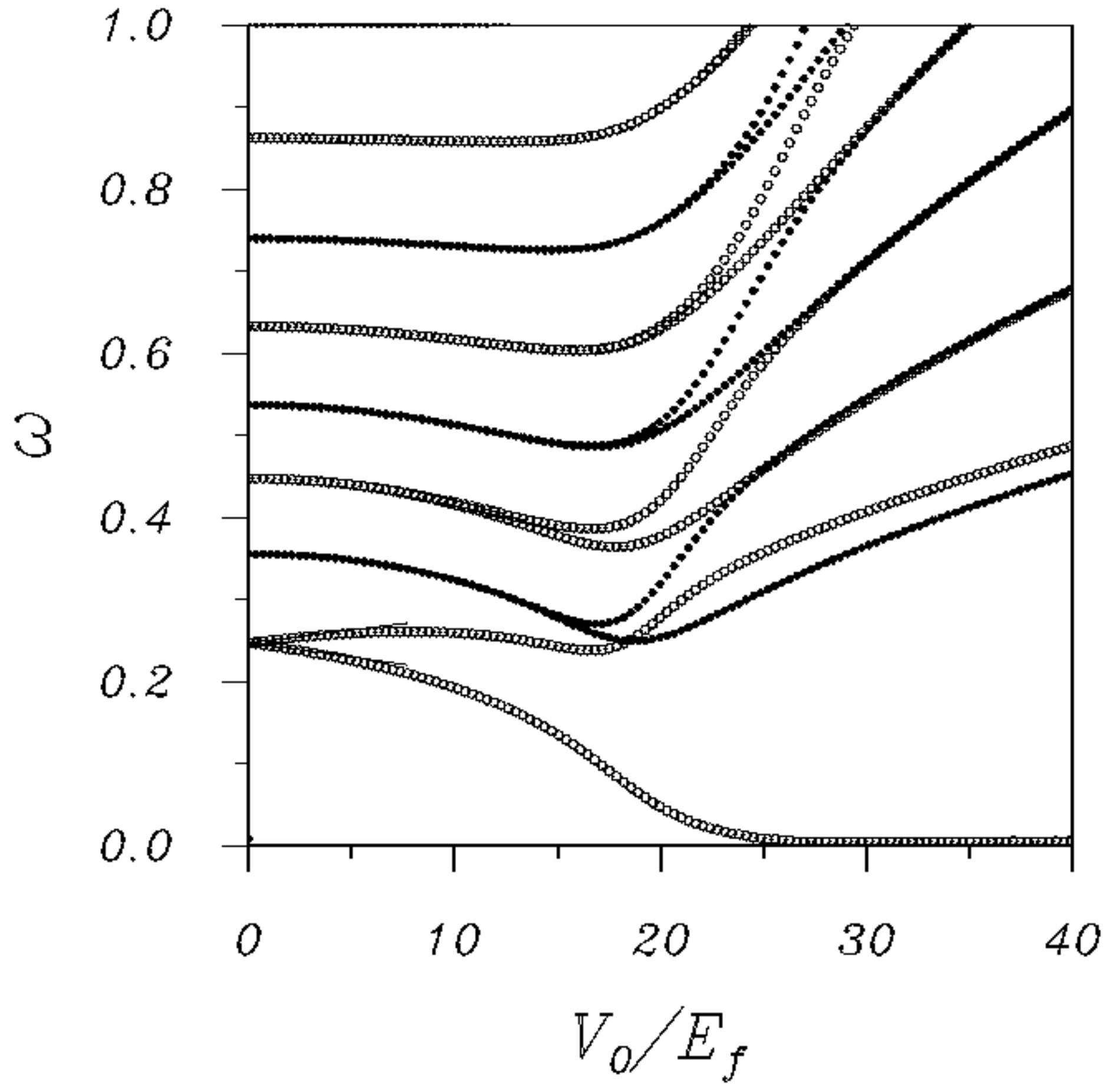


Fig. 15

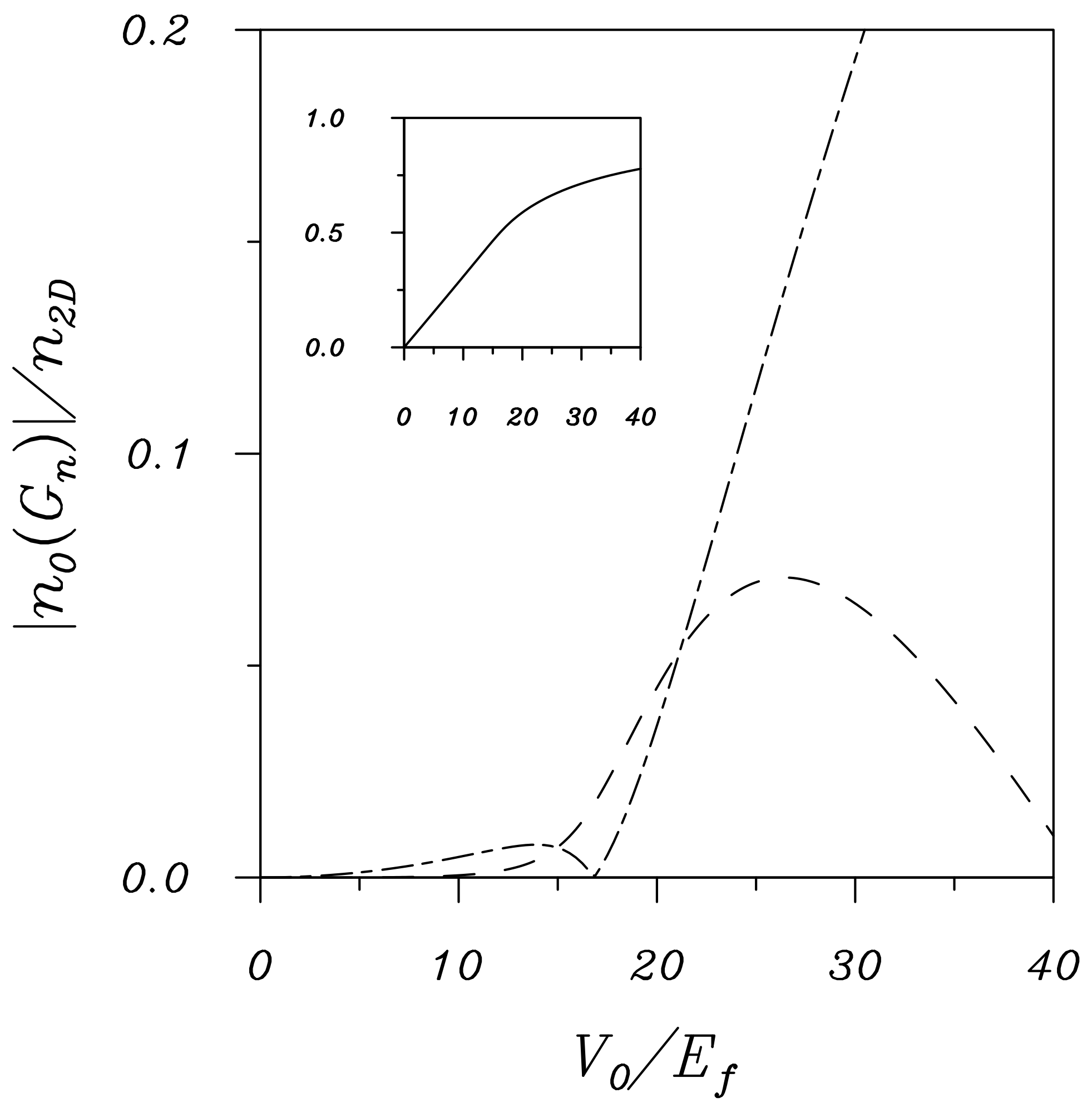

